\title{
An intramolecular theory of the mass-independent isotope effect for ozone. I.
}

\author{
B. C. Hathorn and R. A. Marcus ${ }^{\text {a) }}$ \\ Noyes Laboratories, 127-72, California Institute of Technology, Pasadena, California 91125
}

(Received 23 March 1999; accepted 8 June 1999)

\begin{abstract}
An intramolecular theory of the unusual mass-independent isotope effect for ozone formation and dissociation is described. The experiments include the enrichment factor, its dependence on the ambient pressure, the ratio of the formation rates of symmetric and asymmetric ozone isotopomers, the enrichment of ozone formed from heavily enriched oxygen isotopes, the comparison of that enrichment to that when the heavy isotopes are present in trace amounts, the isotopic exchange rate constant, and the large mass-dependent effect when individual rate constants are measured, in contrast with the mass-independent effect observed for scrambled mixtures. To explain the results it is suggested that apart from the usual symmetry number ratio of a factor of 2, the asymmetric ozone isotopomers have a larger density of reactive (coupled) quantum states, compared with that for the symmetric isotopomers (about 10\%), due to being more "RRKM-like" (Rice-RamspergerKessel-Marcus): Symmetry restricts the number of intramolecular resonances and coupling terms in the Hamiltonian which are responsible for making the motion increasingly chaotic and, thereby, increasingly statistical. As a result the behavior occurs regardless of whether the nuclei are bosons $\left({ }^{16} \mathrm{O},{ }^{18} \mathrm{O}\right)$ or fermions $\left({ }^{17} \mathrm{O}\right)$. Two alternative mechanisms are also considered, one invoking excited electronic states and the other invoking symmetry control in the entrance channel. Arguments against each are given. An expression is given relating the mass-independent rates of the scrambled systems to the mass-dependent rates of the unscrambled ones, and the role played by a partitioning term in the latter is described. Different definitions for the enrichment factor for heavily enriched isotopic systems are also considered. In the present paper attention is focused on setting up theoretical expressions and discussing relationships. They provide a basis for future detailed calculations. (C) 1999 American Institute of Physics.
\end{abstract}

[S0021-9606(99)01433-6]

\section{INTRODUCTION}

Since its discovery in the oxygen in meteorites in $1973^{1}$ and in the laboratory in $1983,{ }^{2}$ mass-independent isotopic enrichment has been the subject of numerous studies. These studies, and others related to enrichment, have been made in ozone in the atmosphere, ${ }^{3-12}$ the laboratory, ${ }^{13-35}$ in other molecules, ${ }^{28,36-39}$ and in ions. ${ }^{40-43}$ In this unusual effect in a chemical reaction, the enrichment or depletion of two isotopes relative to that of a third is equal. In the very common and well known mass-dependent effect, the enrichment $\delta$ is, instead, proportional to the mass difference $\Delta m$ for the isotopic substitution, and thus the normal mass-dependent relative enrichment for ${ }^{17} \mathrm{O}$ and ${ }^{18} \mathrm{O}$, denoted by ${ }^{17} \delta /{ }^{18} \delta$ for ozone, would be $\frac{1}{2}$, instead of unity. ${ }^{44-46}$

A number of authors have suggested symmetry, or excited electronic states, among others, as possible causes of the effect. ${ }^{2,29,36,40,41,47-49}$ For example, trace amounts of ${ }^{17} \mathrm{O}$ or ${ }^{18} \mathrm{O}$, an isotope denoted by $\mathrm{Q}$, have in common that they alone can form the asymmetric molecule QOO, in addition to the symmetric one OQO, whereas the reaction of ${ }^{16} \mathrm{O}$ with ${ }^{32} \mathrm{O}_{2}$ can only form a symmetric species, OOO. It is not merely that QOO has a symmetry number of one, while OOO and OQO have symmetry numbers of two, since such statistical factors are tacitly accounted for in the standard

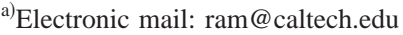

literature definition of $\delta$. What is involved, instead, is a difference in the behavior of OOO and OQO, compared with QOO, which is beyond a mere difference in symmetry number. The latter point has been occasionally overlooked in the literature, whenever it was assumed that the symmetry number of two can be used as a source of the enrichment $\delta{ }^{50}$

For the photodissociation of ozone the current experimental results are not definitive as to the extent of the massdependent vs mass-independent effect and we do not discuss them here. Further, if no long-lived vibrationally excited ozone occurs in the photodissociation, the reaction mechanism would differ dynamically from that considered in the present paper.

There are a variety of experimental results which should be explained: First is the surprising existence of the effect itself. Again, the effect vanishes at higher pressures of the ambient gas or when the role of surfaces is presumed to be important. ${ }^{16}$ At the higher pressures the effect is replaced by a small mass-dependent depletion of the heavier isotopes, instead of the mass-independent enrichment. These "higher pressures" are somewhat below the pressures where the "third-body" fall-off behavior occurs for ozone formation or dissociation. ${ }^{51}$ The mass-independent isotope effect also decreases with decreasing temperature. ${ }^{20}$ Again, the difference $R$ between the observed ratio of QOO/OQO (studied for $\mathrm{Q}={ }^{18} \mathrm{O}$ ) and its statistical value of two is nonzero. A second 
item considered here is the possible relation between $R$ and $\delta$.

Almost all the studies on the mass-independent isotope effect have been with the isotopes ${ }^{17} \mathrm{O}$ and ${ }^{18} \mathrm{O}$ being present in trace amounts. However, there have been several studies with heavy enrichment in the two isotopes: In a study of substantially enriched but scrambled oxygen mixtures of ${ }^{16} \mathrm{O},{ }^{17} \mathrm{O}$, and ${ }^{18} \mathrm{O}$, certain symmetry effects stand out: The most enriched asymmetric ozone isotopomer formed is the one containing all three isotopes 16,17 , and 18 , followed by those which have two atoms of one isotope and one of another. ${ }^{20,23}$ The two ozone molecules consisting of only a single isotope show, instead, relative to ${ }^{16} \mathrm{O}^{16} \mathrm{O}^{16} \mathrm{O}$ a small mass-dependent depletion. The enrichment (depletion) of ${ }^{17} \mathrm{O}^{17} \mathrm{O}^{17} \mathrm{O}$ is intermediate between that of ${ }^{18} \mathrm{O}^{18} \mathrm{O}^{18} \mathrm{O}$ and ${ }^{16} \mathrm{O}^{16} \mathrm{O}^{16} \mathrm{O}$, even though the ${ }^{17} \mathrm{O}$ nuclei are fermions and the ${ }^{16} \mathrm{O}$ and ${ }^{18} \mathrm{O}$ are bosons. The experiments with scrambled mixtures also show that among the six possible isotopic stoichiometric compositions $i j j$ of molecules formed $(i \neq j)$ the enrichments were not exactly equal, but showed some scatter well beyond the experimental errors of $1 \% .^{23}$ They showed, nevertheless, on the average about $\frac{2}{3}$ of the enrichment of that for the ijk molecule where $i, j$, and $k$ are all different.

These studies may be contrasted with those made with heavily enriched but unscrambled mixtures of oxygen. A systematic study of selected reaction pairs showed a massdependent trend. ${ }^{21,22}$ One aim in the present paper is to see how the observed mass-independent behavior of the scrambled mixtures may be consistent with the behavior of the unscrambled ones.

In addition to the mass-independent effect observed for the formation of ozone from the reaction of oxygen molecules with oxygen atoms (formed usually by photolysis ${ }^{13-23}$ but alternatively by pulse radiolysis ${ }^{27}$ or an electric ${ }^{2,20,29}$ or microwave $\mathrm{e}^{30-32}$ discharge), it has also been observed for the thermal decomposition of ozone. ${ }^{33-35}$ Interestingly enough, the thermal dissociation of ozone yields an enrichment of the heavier isotopes, while the formation of ozone also yields this enrichment. That is, in both directions there is enrichment. It might be suggested that two different phenomena are involved. The two experiments do differ in that the dissociation involves a thermal (collisional) activation step of the ozone, while the recombination involves the recombination as the "activation step." To anticipate the results, it will be seen nevertheless that a single mechanism suffices, but that the thermal dissociation results may be complicated, as was indicated by the authors of that work, by surface effects. $^{35}$

In the present article we address these and related questions. The principal idea in this paper is to use RRKM theory, and in particular to use its statistical assumption for the quantum states of the energetic molecule, as the zerothorder approximation. We then treat a correction to the density of states $\rho$ of the symmetric molecule as a small perturbation, $\sim 10 \%$ in the present instance. The reason for distinguishing between the $\rho$ 's of symmetric and asymmetric molecules in this respect is described in Sec. V.

The RRKM recombination and dissociation rate con- stants, for the case of only one entrance-exit channel, can be written as ${ }^{52-58}$

$k_{\mathrm{rec}}=\omega \sum_{J K} \int_{E=0}^{\infty} \frac{\zeta(E J K) N^{\dagger}(E J K)}{\left(N^{\dagger} / h \rho\right)+\omega} \frac{e^{-E / k_{B} T} d E}{h Q^{(1,2)}}$,

$k_{\mathrm{diss}}=\omega e^{-\Delta U / k_{B} T} \sum_{J K} \int_{E=0}^{\infty} \frac{\zeta(E J K) N^{\dagger}(E J K)}{\left(N^{\dagger} / h \rho\right)+\omega} \frac{e^{-E / k_{B} T} d E}{h Q^{(3)}}$,

where $J$ is the total angular momentum, $K$ is any approximately conserved quantum number along the reaction coordinate, and there may be no $K, N^{\dagger}(E J K)$ is the number of quantum states of the transition state, $\zeta$ depends on $E J K$ and equals unity when the system can surmount the barrier, including the zero-point energy of the transition state, and is zero otherwise, $\rho$ is the density of reactive quantum states of the energetic molecule, $\omega$ is a deactivation collision frequency, $\Delta U$ is the change of potential energy for the triatom $\rightarrow$ atom + diatom reaction, and $Q^{(1,2)}$ and $Q^{(3)}$ are the partition functions of the atom-diatom pair and the triatomic molecule, respectively, each in the center-of-mass system of coordinates. Each $Q$ contains the zero-point energy of the reacting pair or the dissociating molecule.

Nowadays in RRKM theory the position of the microcanonical transition state is frequently determined variationally. The rotations in the reacting pair become hindered rotations in the transition state and become bending vibrations in the molecule (and vice versa for unimolecular dissociation) ${ }^{55-63}$ This effect contributes to the effective barrier of the forward and the reverse reaction, and the $\zeta$ in the integrand is zero unless the total barrier is overcome. The transition state is determined where $N^{\dagger}(E J K)$, as a function of a suitable reaction coordinate, is a minimum, i.e., occurs at an entropic bottleneck in a microcanonical system. Finding the most suitable reaction coordinate (and then the position of the transition state along it) has been an interesting problem addressed in recent years for activationless bimolecular recombination reactions and for the reverse unimolecular dissociations. ${ }^{59}$

The density of states $\rho$ and its possible difference for symmetric and asymmetric molecules, apart from the usual symmetry number, and how that difference is expected to depend on pressure at pressures far lower than the "fall-off region" in the unimolecular reaction, will be a particular theme in the present paper, as will the difference in behavior of the scrambled and unscrambled mixtures. At these pressures, the $\omega$ term in the denominator can be neglected, and these equations become

$$
\begin{aligned}
& k_{\mathrm{rec}}=\omega \sum_{J K} \int_{E=0}^{\infty} \zeta \rho e^{-E / k_{B} T} d E / Q^{(1,2)}, \\
& k_{\mathrm{diss}}=\omega e^{-\Delta U / k_{B} T} \sum_{J K} \int_{E=0}^{\infty} \zeta \rho e^{-E / k_{B} T} d E / Q^{(3)} .
\end{aligned}
$$

In Eqs. (1.1) and (1.2) a single deactivation collision frequency $\omega$ was used. Actually, the deactivation can be energy dependent, and so an expression which allows for this dependence and which, correspondingly, is more complicated than Eqs. (1.1) and (1.2) is sometimes used. ${ }^{54-56,64-69}$ However, at low pressures it reduces, in effect, to Eqs. (1.3) 
and (1.4), where $\omega$ is now the deactivation collision frequency for energetic molecules whose energy is just above the threshold energy. ${ }^{64-66}$

For Eqs. (1.1) and (1.2) there is only one entrance-exit channel. However, for the reactions in this paper, there are two exit channels $a$ and $b$, as in $\mathrm{QOO}^{*} \rightarrow \mathrm{Q}+\mathrm{O}_{2}$ and $\mathrm{QOO}^{*} \rightarrow \mathrm{QO}+\mathrm{O}$, where $\mathrm{Q}$ denotes an ${ }^{17} \mathrm{O}$ or ${ }^{18} \mathrm{O}$ isotope, and the asterisk denotes a vibrationally excited molecule. In this case, it is shown in Appendix A how the equations are modified: If $N_{a}^{\dagger}$ and $N_{b}^{\dagger}$ denote the number of quantum states of the transition state in each exit channel, one obtains Eqs. (1.5)-(1.9), instead of Eqs. (1.1)-(1.4), for the reaction leading to or coming from channel $a$.

$$
k_{\mathrm{rec}}^{a}=\omega \sum_{J K} \int_{0}^{\infty} \frac{N_{a}^{\dagger} e^{-E / k_{B} T}}{\omega+\left(N_{a}^{\dagger}+N_{b}^{\dagger}\right) / h \rho} \frac{d E}{h Q_{a}^{(1,2)}},
$$

where $Q_{a}^{(1,2)}$ is the partition function for the reactants in the initial channel $a$.

The rate constant for dissociation into channel $a$ is

$$
k_{\mathrm{diss}}^{a}=\omega e^{-\Delta U / k_{B} T} \sum_{J K} \int_{0}^{\infty} \frac{N_{a}^{\dagger} e^{-E / k_{B} T}}{\omega+\left(N_{a}^{\dagger}+N_{b}^{\dagger}\right) / h \rho} \frac{d E}{h Q^{(3)}} .
$$

When $\omega \rightarrow 0$, the $k_{\text {rec }}^{a}$ and $k_{\text {diss }}^{a}$ become

$$
k_{\mathrm{rec}}^{a}=\omega \sum_{J K} \int_{E=0}^{\infty} Y_{a}^{\dagger} \rho e^{-E / k_{B} T} d E / Q_{a}^{(1,2)}, \quad(\omega \rightarrow 0)
$$

and

$$
k_{\mathrm{diss}}^{a}=\omega e^{-\Delta U / k_{B} T} \sum_{J K} \int_{E=0}^{\infty} Y_{a}^{\dagger} \rho e^{-E / k_{B} T} d E / Q^{(3)}, \quad(\omega \rightarrow 0),
$$

where

$$
Y_{a}^{\dagger}=N_{a}^{\dagger} /\left(N_{a}^{\dagger}+N_{b}^{\dagger}\right)
$$

When $\omega \rightarrow \infty$ in Eq. (1.5), we have, instead

$$
k_{\mathrm{rec}}^{a, \infty}=\sum_{J K} \int_{E=0}^{\infty} N_{a}^{\dagger} e^{-E / k_{B} T} d E / h Q_{a}^{(1,2)} .
$$

In these equations it is understood in the integral-sum that the integrand-summand is zero unless the respective barrier is exceeded, namely, such that $N_{a}^{\dagger}$ is equal to or greater than unity, or $N_{b}^{\dagger}$ is greater than or equal to unity in the case of the second channel. That is, the $N_{a}^{\dagger}$ in the numerator of Eqs. $(1.5)-(1.8)$ and (1.10) also plays the role played by $\zeta$ in Eqs. (1.1)-(1.4), and so the $\zeta$ is omitted in Eqs. (1.5)-(1.8) and (1.10).

When both exit channels yield indistinguishable products, $Y_{a}^{\dagger}$ equals $\frac{1}{2}$ exactly and this factor can be absorbed into $\rho$ and regarded as a symmetry number. Accordingly, $\rho_{\mathrm{OOO}}$, $\rho_{\mathrm{OQO}}$, and $\rho_{j i j}$ in the subsequent sections will contain this factor of $\frac{1}{2}$ as a conventional symmetry number 2 . We allow later for the possibility of non-RRKM behavior for the ozone, each $\rho$ then indicating an effective density of quantum states of the energetic molecule.

We shall also need expressions for the exchange rate constant for a reaction where $a$ is the incident channel and $b$ is the exit channel, leading to exchange. The result is derived in Appendix A and is given by

$$
k_{\mathrm{ex}}^{a \rightarrow b}=\frac{1}{h Q_{a}^{(1,2)}} \sum_{J K} \int_{E} \frac{N_{a}^{\dagger} N_{b}^{\dagger}}{h \rho \omega+N_{a}^{\dagger}+N_{b}^{\dagger}} e^{-E / k_{B} T} d E .
$$

At low pressures, this equation reduces to

$$
k_{\mathrm{ex}}^{a}(\omega \rightarrow 0)=\frac{1}{h Q_{a}^{(1,2)}} \sum_{J K} \int N_{a}^{\dagger} Y_{b}^{\dagger} e^{-E / k_{B} T} d E .
$$

A mechanism for the mass-independent effect in ozone formation is described in Sec. II for the most frequently studied case, systems for which the isotopes ${ }^{17} \mathrm{O}$ or ${ }^{18} \mathrm{O}$ are present in trace amounts. The mass-independent effect in the thermal dissociation of ozone is considered in Sec. III. In Sec. IV the formation of ozone is discussed where the initial oxygen is substantially enriched in all three isotopes. Several possible definitions of the enrichment are also considered there. Symmetry-related restrictions on intramolecular coupling as a possible source for a smaller $\rho$ of reactive states for the symmetric molecules are described in Sec. V. Results and data are discussed in Sec. VI, including the role played by the partitioning factor $Y_{a}^{\dagger}$ in the unscrambled mixtures' experiments. A mechanism involving low-lying excited electronic states is considered and excluded, on the basis of angular momentum conservation, as a source of $\delta$ in Appendix B. Symmetry restrictions along the reaction coordinate from reacting pair to the transition state as another source are also considered and the reason for presently discarding that source for the current system is given.

Although the present mechanism is described for the ozone formation and ozone thermal dissociation experiments, the oxygen atoms in the former being produced by the photodissociation (or electric discharge) of molecular oxygen, related arguments can be used for other systems. The arguments are not intended to preclude other mechanisms for certain systems. For example, in photodissociation experiments of ozone a long-lived "activated molecule" may not exist, but rather a more or less direct dissociation step may occur instead, and symmetry effects on the dissociation process at curve crossings of the electronic states have been implicated. $^{47,48}$

We have undertaken numerous calculations based on equations derived in the present paper, and they will be submitted for publication separately.

\section{MECHANISM FOR OZONE FORMATION}

The chemical reactions in the ozone formation when two of the isotopes are present in trace amounts can be summarized as follows:

\begin{tabular}{lc}
\multicolumn{1}{c}{ Reaction step } & Rate constant \\
$\mathrm{O}_{2}+h \nu \rightarrow 2 \mathrm{O}$ & $2 I$, \\
$\mathrm{QO}+h \nu \rightarrow \mathrm{Q}+\mathrm{O}$ & $I$, \\
$\mathrm{O}+\mathrm{O}_{2} \rightarrow \mathrm{O}_{3}$ & $k_{6,66}^{s}$, \\
$\mathrm{O}+\mathrm{QO} \rightarrow \mathrm{QOO}$ & $k_{6, q 6}^{a s}$, \\
$\mathrm{O}+\mathrm{QO} \rightarrow \mathrm{OQO}$ & $k_{6, q 6}^{s}$, \\
$\mathrm{Q}+\mathrm{O}_{2} \rightarrow \mathrm{QOO}$ & $k_{q, 66}^{a s}$, \\
$\mathrm{O}+\mathrm{QO} \rightleftharpoons \mathrm{Q}+\mathrm{O}_{2}$ & $\left(K_{\mathrm{ex}}\right)$,
\end{tabular}

where we have introduced a notation for the rate constants to conform with that used for the more general system of 
heavily enriched isotopes treated in Sec. IV. The as and $s$ superscripts denote the formation of asymmetric and symmetric ozones $\mathrm{QO}_{2}$. The $K_{\text {ex }}$ denotes the equilibrium constant of reaction (2.7). We have supposed in the first two steps that the $\mathrm{O}_{2}$ and QO have the same integrated absorption coefficients and quantum yields for formation of atoms. The $I$ in Eqs. (2.1) and (2.2) denotes a "rate constant" for the formation of oxygen atoms and is proportional to the light intensity.

Since the exchange rate constants for forward and reverse steps in Eq. (2.7) are several orders of magnitude larger than the recombination rate constants $k$ in Eqs. (2.3)-(2.6) at the prevailing pressures ${ }^{70-72}$ (values of rate constants are given later), a local equilibrium exists for reaction (2.7)

$\mathrm{Q} \cdot \mathrm{O}_{2} / \mathrm{O} \cdot \mathrm{QO}=K_{\mathrm{ex}} \cdot$

The formation rates of the various ozone molecules are

$$
\begin{aligned}
& d \mathrm{O}_{3} / d t=k_{6,66}^{s} \mathrm{O} \cdot \mathrm{O}_{2}, \quad d \mathrm{OQO} / d t=k_{6, q 6}^{s} \mathrm{O} \cdot \mathrm{QO}, \\
& d \mathrm{QOO} / d t=k_{6, q 6}^{a s} \mathrm{O} \cdot \mathrm{QO}+k_{q, 66}^{a s} \mathrm{Q} \cdot \mathrm{O}_{2} \\
& =\left(k_{6, q 6}^{a s}+k_{q, 66}^{a s} K_{\mathrm{ex}}\right) \mathrm{O} \cdot \mathrm{QO},
\end{aligned}
$$

and so the ratio of reaction yields is

$$
\frac{\mathrm{QOO}+\mathrm{OQO}}{\mathrm{O}_{3}}=\frac{\mathrm{QO}}{\mathrm{O}_{2}} \frac{\left(k_{6, q 6}^{s}+k_{6, q 6}^{a s}+k_{q, 66}^{a s} K_{\mathrm{ex}}\right)}{k_{6,66}^{s}} .
$$

The standard definition of the enrichment, conventionally denoted by $\delta$ but normally multiplied by 1000 , is the $\mathrm{Q} / \mathrm{O}$ ratio in the ozone product divided by the $\mathrm{Q} / \mathrm{O}$ ratio in the reactants, minus $1 .^{44,46}$ So defined, $\delta$ equals zero in the purely statistical case. In the present case it becomes

$$
\delta=\frac{[\mathrm{QOO}+\mathrm{OQO}] / 3 \mathrm{O}_{3}}{\mathrm{QO} / 2 \mathrm{O}_{2}}-1 .
$$

Thereby, we have from Eqs. (2.9)-(2.12)

$$
\delta=\frac{2\left(k_{6, q 6}^{s}+k_{6, q 6}^{a s}+k_{q, 66}^{a s} K_{\mathrm{ex}}\right)}{3 k_{6,66}^{s}}-1 .
$$

A second quantity of interest is the ratio QOO/OQO. The difference $R$ from its statistical value of 2 is obtained from Eqs. (2.9) $-(2.10)$

$$
\begin{aligned}
R=\frac{\mathrm{QOO}}{\mathrm{OQO}}-2 & =\frac{k_{6, q 6}^{a s}+k_{q, 66}^{a s} K_{\mathrm{ex}}}{k_{6, q 6}^{s}}-2 \\
& =3 \delta\left(\frac{k_{6,66}^{s}}{2 k_{6, q 6}^{s}}\right)+\frac{3\left(k_{6,66}^{s}-2 k_{6, q 6}^{s}\right)}{2 k_{6, q 6}^{s}} .
\end{aligned}
$$

We later compare this relationship of $R$ and $\delta$ with the currently available experimental results. ${ }^{17-19}$ When $k_{6,66}^{s}$ $\cong 2 k_{6, q 6}^{s}$, i.e., when this mass-dependent effect is sufficiently small, the right hand side of Eq. (2.14) equals $3 \delta$.

We consider next the rate constants in terms of the recombination rate constant expressions in Sec. I. For the low ambient pressures of interest here, the vibrationally energetic molecules almost entirely redissociate rather than being deactivated by collision. Thus, Eq. (1.7) is the one to be used. We obtain

$$
\begin{aligned}
& k_{6,66}^{s}=\omega \sum \int \zeta_{\mathrm{OOO}} \rho_{\mathrm{OOO}} e^{-E / k_{B} T} d E / Q_{\mathrm{O}, \mathrm{OO}}, \\
& k_{6, q 6}^{s}=\omega \sum \int \zeta_{\mathrm{OQO}} \rho_{\mathrm{OQO}} e^{-E / k_{B} T} d E / Q_{\mathrm{O}, \mathrm{QO}}, \\
& k_{6, q 6}^{a s}=\omega \sum \int Y_{\mathrm{O}, \mathrm{QO}}^{\dagger} \rho_{\mathrm{QOO}} e^{-E / k_{B} T} d E / Q_{\mathrm{O}, \mathrm{QO}}, \\
& k_{q, 66}^{a s} K_{\mathrm{ex}}=\omega \sum \int Y_{\mathrm{Q}, \mathrm{OO}}^{\dagger} \rho_{\mathrm{QOO}} e^{-E / k_{B} T} d E / Q_{\mathrm{O}, \mathrm{QO}},
\end{aligned}
$$

where the $Q$ 's denote the partition functions of the cited pairs, in the center-of-mass system of coordinates in each case. For the reasons discussed later, the $\rho_{\mathrm{OOO}}$ and $\rho_{\mathrm{OQO}}$ but not $\rho_{\mathrm{QOO}}$ may vary from a value denoted by $\rho_{\mathrm{OOO}}^{s}$ and $\rho_{\mathrm{OQO}}^{s}$ at low pressures to a value $\rho_{\mathrm{OOO}}^{a s}$ and $\rho_{\mathrm{OQO}}^{a s}$ at higher pressures, with $\rho^{a s}>\rho^{s}$. The $\rho_{\text {QOO }}$ in Eqs. (2.17) and (2.18), on the other hand, remains relatively unchanged with pressure. The $\rho_{\mathrm{OOO}}$ and $\rho_{\mathrm{OQO}}$ in Eq. (2.15) each contain a symmetry number of two in their respective denominators, as discussed in Sec. I, and $Q_{\mathrm{O}, \mathrm{OO}}$ does also. The $\omega$ 's in Eqs. (2.15)-(2.18) may differ slightly and these differences will, throughout this paper, be absorbed in the relevant $\rho / Q$ 's, for notational brevity, but will be included in any future actual calculation.

In Eq. (2.17) $Y_{\mathrm{O}, \mathrm{QO}}^{\dagger}$ denotes the factor $N_{a}^{\dagger} /\left(N_{a}^{\dagger}+N_{b}^{\dagger}\right)$ appearing in Eq. (1.7), the $a$ and $b$ denoting the $\mathrm{O}+\mathrm{QO}$ and $\mathrm{Q}+\mathrm{O}_{2}$ entrance channels into $\mathrm{QOO}^{*}$, respectively. The relation $K_{\mathrm{ex}}=Q_{\mathrm{Q}, \mathrm{O}_{2}} / Q_{\mathrm{O}, \mathrm{QO}}$ was introduced into the left-hand side of Eq. (2.18).

From Eqs. (2.11) and (2.15)-(2.18) it follows that:

$$
\begin{aligned}
& \frac{\mathrm{OQO}+\mathrm{OOO}}{\mathrm{O}_{3}} \\
& =\frac{\sum \int\left(\zeta_{\mathrm{QOO}} \rho_{\mathrm{QOO}}+\zeta_{\mathrm{OQO}} \rho_{\mathrm{OQO}}\right) e^{-E / k_{B} T} d E / Q_{\mathrm{O}, \mathrm{QO}}}{\sum \int \zeta_{\mathrm{OOO}} \rho_{\mathrm{OOO}} e^{-E / k_{B} T} d E / Q_{\mathrm{O}, \mathrm{OO}}} .
\end{aligned}
$$

Upon looking at the origin of Eq. (2.19), one sees that the $\zeta_{\mathrm{QOO}}$ is zero until the smaller of $N_{\mathrm{OQO}}^{\dagger}$ and $N_{\mathrm{QOO}}^{\dagger}$ becomes equal to or exceeds unity, while the $\zeta_{\mathrm{OQO}}$ is zero until $N_{\mathrm{OQO}}^{\dagger}$ becomes equal to or exceeds unity. From Eqs. (2.12) and (2.19) we have

$$
\delta=\frac{2}{3} \frac{\sum \int\left(\zeta_{\mathrm{QOO}} \rho_{\mathrm{QOO}}+\zeta_{\mathrm{OQO}} \rho_{\mathrm{OQO}}\right) e^{-E / k_{B} T} d E / Q_{\mathrm{O}, \mathrm{QO}}}{\sum \int \zeta_{\mathrm{OOO}} \rho_{\mathrm{OOO}} e^{-E / k_{B} T} d E / Q_{\mathrm{O}, \mathrm{OO}}}-1 .
$$

When a presumably small mass-dependent effect is neglected, the ratio $\Sigma \int \zeta_{\mathrm{OOO}} \rho_{\mathrm{OOO}} \exp \left(-E / k_{B} T\right) d E / 2 Q_{\mathrm{O}, \mathrm{OO}}$ can be equated to $\Sigma \int \zeta_{\mathrm{OQO}} \rho_{\mathrm{OQO}} \exp \left(-E / k_{B} T\right) d E / Q_{\mathrm{O}, \mathrm{QO}}$, the factor of 2 compensating for the ratio of symmetry numbers in $Q_{\mathrm{O}, \mathrm{OO}} / Q_{\mathrm{O}, \mathrm{QO}}$. Further, if the transition state is loose, the $\zeta_{\mathrm{OQO}}$ is zero unless the zero-point energy of QO (plus other terms) is exceeded, an effect which tends to cancel the effect of that zero-point energy in $Q_{\mathrm{O}, \mathrm{QO}}$. A similar remark applies to $\zeta_{\mathrm{OOO}}$ and $Q_{\mathrm{O}, \mathrm{OO}}$.

We would then have

$$
\delta=\frac{2 \sum \int\left(\zeta_{\mathrm{QOO}} \rho_{\mathrm{QOO}}-2 \zeta_{\mathrm{OQO}} \rho_{\mathrm{OQO}}\right) e^{-E / k_{B} T} d E / Q_{\mathrm{O}, \mathrm{QO}}}{3 \sum \int \zeta_{\mathrm{OOO}} \rho_{\mathrm{OOO}} e^{-E / k_{B} T} d E / Q_{\mathrm{O}, \mathrm{OO}}} .
$$


The $\zeta_{\mathrm{QOO}} \rho_{\mathrm{QOO}}-2 \zeta_{\mathrm{OQO}} \rho_{\mathrm{OQO}}$ in the numerator is the extra density of states, assumed in Sec. V to be due to somewhat fewer restrictions on intramolecular coupling in asymmetric molecules.

If we introduce the following quantities, $k_{a s}$ and $k_{s}$ for the recombination rate constants for the asymmetric and symmetric species

$$
\begin{aligned}
k_{a s} & =\omega \sum \int \zeta_{\mathrm{QOO}} \rho_{\mathrm{QOO}} e^{-E / k_{B} T} d E / Q_{\mathrm{O}, \mathrm{QO}}, \\
k_{s} & =\omega \sum \int \zeta_{\mathrm{OOO}} \rho_{\mathrm{OOO}} e^{-E / k_{B} T} d E / Q_{\mathrm{O}, \mathrm{OO}} \\
& \simeq \omega \sum \int 2 \zeta_{\mathrm{OQO}} \rho_{\mathrm{OQO}} e^{-E / k_{B} T} d E / Q_{\mathrm{O}, \mathrm{QO}} .
\end{aligned}
$$

Equation (2.21) becomes

$$
\delta=\frac{2}{3} \frac{k_{a s}-k_{s}}{k_{s}} .
$$

At very low pressures $k_{s}$ is perhaps about $0.9 k_{a s}$, judging from the value ${ }^{16}$ of $\delta$, while at higher pressures, where the mass-independent effect disappears, as discussed in Sec. V, $k_{s} \simeq k_{a s}$.

\section{THERMAL DECOMPOSITION OF OZONE}

Mass-independent enrichment of the heavier isotopes has also been found in the thermal dissociation of ozone. ${ }^{33-35}$ In the usual reaction scheme the $\mathrm{O}_{3}$ dissociates to $\mathrm{O}+\mathrm{O}_{2}$, followed by the reaction $\mathrm{O}+\mathrm{O}_{3} \rightarrow 2 \mathrm{O}_{2}$. The competing reaction for the disappearance of $\mathrm{O}, \mathrm{O}+\mathrm{O}_{2}+\mathrm{M} \rightarrow \mathrm{O}_{3}+\mathrm{M}$, where $\mathrm{M}$ is a third body, is estimated to be only several percent of the above reaction for $\mathrm{O}$ disappearance. ${ }^{70}$

We use the following reaction scheme for systems where the isotopes $\mathrm{Q}$ are present in trace amounts:

\begin{tabular}{lc}
\multicolumn{1}{c}{ Reaction step } & Rate constant \\
\cline { 1 - 1 } $\mathrm{O}_{3} \rightarrow \mathrm{O}+\mathrm{O}_{2}$ & $k_{\mathrm{OOO}}^{s}$, \\
$\mathrm{QOO} \rightarrow \mathrm{Q}+\mathrm{O}_{2}$ & $k_{\mathrm{QOO}}^{a s}$, \\
$\mathrm{QOO} \rightarrow \mathrm{O}+\mathrm{QO}$ & $k_{\mathrm{OQO}}^{s}$, \\
$\mathrm{OQO} \rightarrow \mathrm{O}+\mathrm{QO}$ & $k_{3}$, \\
$\mathrm{O}+\mathrm{O}_{3} \rightarrow 2 \mathrm{O}_{2}$ & $k_{4}$, \\
$\mathrm{Q}+\mathrm{O}_{3} \rightarrow \mathrm{QO}+\mathrm{O}_{2}$ &
\end{tabular}

where we have omitted the reactions of $\mathrm{O}$ with $\mathrm{QOO}$ and OQO, which are small relative to the reaction of $\mathrm{O}$ with $\mathrm{O}_{3}$. The exchange reactions, Eqs. (2.7) and (2.8), are also included: Even though oxygen is a reaction product, the percent conversions were $10 \%$ or more and the exchange rate constants are about 400 fold greater than the recombination rate constants $k_{3}$ or $k_{4},{ }^{70,71}$ and so the exchange reactions must be included. The $k_{\mathrm{QOO}}^{a s}$ denotes the sum of the rate constants of reactions (3.2) and (3.3).

Every Q formed in reaction (3.2) ultimately forms a QO, either via an exchange reaction with $\mathrm{O}_{2}$ or via reaction (3.6). Thus,

$$
\frac{d \mathrm{QO}}{d t}=k_{\mathrm{QOO}}^{a s} \mathrm{QOO}+k_{\mathrm{OQO}}^{s} \mathrm{OQO} .
$$

The ratio $\mathrm{QOO} /\left(\mathrm{QO}_{2}\right)_{\text {tot }}$, where $\left(\mathrm{QO}_{2}\right)_{\text {tot }}$ denotes $\mathrm{QOO}+\mathrm{OQO}$, for the initial equilibrated (scrambled) ozone, equals the ratio of the partition functions $Q_{\mathrm{QOO}} /\left(Q_{\mathrm{QOO}}\right.$ $+Q_{\mathrm{OQO}}$ ), and so Eq. (3.7) yields

$$
\frac{d \mathrm{QO}}{d t}=\frac{k_{\mathrm{QOO}}^{a s} Q_{\mathrm{QOO}}+k_{\mathrm{OQO}}^{s} Q_{\mathrm{OQO}}}{\left(Q_{\mathrm{QOO}}\right)_{\mathrm{tot}}} \quad\left(\mathrm{QO}_{2}\right)_{\mathrm{tot}},
$$

where

$$
\left(Q_{\mathrm{QOO}}\right)_{\mathrm{tot}}=Q_{\mathrm{QOO}}+Q_{\mathrm{OQO}} .
$$

Essentially all the $\mathrm{O}_{2}$ is formed, when $\mathrm{Q}$ is in trace amounts, by reactions (3.1) and (3.5), and so

$$
\frac{d \mathrm{O}_{2}}{d t} \simeq 3 k_{\mathrm{OOO}}^{s} \mathrm{O}_{3} .
$$

The ratio of $\mathrm{QO} / \mathrm{O}_{2}$ formed is thus

$$
\frac{\mathrm{QO}}{\mathrm{O}_{2}}=\frac{\left(k_{\mathrm{QOO}}^{a s} Q_{\mathrm{QOO}}+k_{\mathrm{OQO}}^{s} Q_{\mathrm{OQO}}\right)}{k_{\mathrm{OOO}}^{s}\left(Q_{\mathrm{QOO}}\right)_{\mathrm{tot}}} \frac{\left(\mathrm{QO}_{2}\right)_{\mathrm{tot}}}{3 \mathrm{O}_{3}} .
$$

The $\mathrm{Q} / \mathrm{O}$ ratio in the reactants is $\left(\mathrm{QO}_{2}\right)_{\text {tot }} / 3 \mathrm{O}_{3}$, and so $\delta$ equals $\left\{\left(\mathrm{QO} / \mathrm{O}_{2}\right) /\left[\left(\mathrm{QO}_{2}\right)_{\mathrm{tot}} / 3\right]\right\} \mathrm{O}_{3}-1$. We thus have

$$
\delta=\frac{k_{\mathrm{QOO}}^{a s} Q_{\mathrm{QOO}}+k_{\mathrm{OQO}}^{s} Q_{\mathrm{OQO}}}{k_{\mathrm{OOO}}^{s}\left(Q_{\mathrm{QOO}}\right)_{\mathrm{tot}}}-1 \text {. }
$$

We next introduce the expressions of Sec. I for the unimolecular dissociation rate constants. We have

$$
\delta=\frac{\sum \int\left(\zeta_{\mathrm{QOO}} \rho_{\mathrm{QOO}}+\zeta_{\mathrm{OQO}} \rho_{\mathrm{OQO}}\right) e^{-E / k_{B} T} d E /\left(Q_{\mathrm{QOO}}\right)_{\mathrm{tot}}}{\sum \int \zeta_{\mathrm{OOO}} \rho_{\mathrm{OOO}} e^{-E / k_{B} T} d E / Q_{\mathrm{OOO}}}-1,
$$

where a factor $\exp \left(-\Delta U / k_{B} T\right)$ in numerator and denominator has been cancelled. This expression differs from that in Eq. (2.20) by a factor $3 K / 2$, where

$$
K=\frac{Q_{\mathrm{OOO}}}{\left(Q_{\mathrm{QOO}}\right)_{\text {tot }}} \frac{Q_{\mathrm{O}, \mathrm{QO}}}{Q_{\mathrm{O}, \mathrm{OO}}} .
$$

It is readily shown that this $K$ is the equilibrium constant for the reaction

$$
\left(\mathrm{QO}_{2}\right)_{\text {tot }}+\mathrm{O}_{2} \rightleftharpoons \mathrm{O}_{3}+\mathrm{QO} \text {, }
$$

as follows: The equilibrium constant of Eq. (3.14) equals $Q_{\mathrm{OOO}}^{\prime} Q_{\mathrm{QO}}^{\prime} /\left(Q_{\mathrm{QO}_{2}}^{\prime}\right)_{\text {tot }} Q_{\mathrm{OO}}^{\prime}$, where the primes indicate that the respective translational partition functions are now included. If we multiply this ratio by $Q_{\mathrm{O}}^{\prime} / Q_{\mathrm{O}}^{\prime}$ and then convert the product of partition functions of each atom-diatom pair to one involving the center-of-mass of the pair and one involving their relative motion, the equilibrium constant of Eq. (3.14) becomes identical to the $K$ given by Eq. (3.13). The value of $K$ is close to $\frac{2}{3}$.

\section{OZONE FORMATION FROM HEAVILY ENRICHED OXYGEN}

We consider next the experiments in which there are arbitrarily large or small amounts of each of the 16,17, and 18 isotopes, ${ }^{15,17,19,23,32}$ and introduce symbols ${ }^{i} \mathrm{O}$, where $i$ $=6,7,8$, to denote oxygen atoms of mass 16, 17, and 18, respectively, and ${ }^{i j} \mathrm{O}_{2}$ to denote oxygen molecules having 
isotopes $i$ and $j$. Typical steps in the reaction sequence for the photolysis of oxygen are

$\begin{array}{ll}\frac{\text { Reaction step }}{{ }^{i j} \mathrm{O}_{2}+h \nu \rightarrow{ }^{i} \mathrm{O}+{ }^{j} \mathrm{O},} & \text { Rate constant } \\ { }^{i} \mathrm{O}+{ }^{j k} \mathrm{O}_{2} \rightarrow{ }^{i j k} \mathrm{O}_{3} & k_{i, j k}^{s} \text { or } k_{i, j k}^{a s}, \\ { }^{i} \mathrm{O}+{ }^{j k} \mathrm{O}_{2} \rightleftharpoons{ }^{k} \mathrm{O}+{ }^{i j} \mathrm{O}_{2} & \left(K_{i, j k}^{k, i j}\right),\end{array}$

where ${ }^{i j k} \mathrm{O}_{3}$ and ${ }^{i k j} \mathrm{O}_{3}$, but not ${ }^{i j} \mathrm{O}_{2}$ and ${ }^{j i} \mathrm{O}_{2}$, are distinguishable, and where a rapid exchange reaction (4.3) establishes a local equilibrium whose equilibrium constant is $K_{i, j k}^{k, i j}$. The $s$ or as label in Eq. (4.2) is again used to denote the formation of a symmetric or asymmetric ozone molecule.

We first consider the enrichment factor for the ijj ozone molecules. For forming ${ }^{i j j} \mathrm{O}_{3}$ and ${ }^{j i j} \mathrm{O}_{3}$ we have in the steady-state

$$
\begin{aligned}
d\left({ }^{i j j} \mathrm{O}_{3}\right)_{\mathrm{tot}} / d t & =\omega\left({ }^{i j j} \mathrm{O}_{3}^{*}\right)_{\mathrm{tot}} \\
& =k_{i, j j}^{a s}{ }^{i} \mathrm{O} \cdot{ }^{j j} \mathrm{O}_{2}+\left(k_{j, i j}^{a s}+k_{j, i j}^{s}\right){ }^{j} \mathrm{O} \cdot{ }^{i j} \mathrm{O}_{2},
\end{aligned}
$$

where $\left({ }^{i j j} \mathrm{O}_{3}\right)$ tot denotes ${ }^{i j j} \mathrm{O}_{3}+{ }^{j i j} \mathrm{O}_{3}$. Because of the fast exchange reactions (4.3) an equilibrium constant $K_{i, i j}^{i, j j}$, associated with the inverse of reaction (4.3) for $k=j$, can be introduced to relate the instantaneous concentrations

$$
{ }^{i} \mathrm{O} \cdot{ }^{j j} \mathrm{O}_{2} /{ }^{j} \mathrm{O} \cdot{ }^{i j} \mathrm{O}_{2}=K_{j, i j}^{i, j j},
$$

and used to convert the product ${ }^{i} \mathrm{O} \cdot{ }^{j j} \mathrm{O}_{2}$ in Eq. (4.4) to ${ }^{j} \mathrm{O} \cdot{ }^{i j} \mathrm{O}_{2}$. We then obtain from Eq. (4.4)

$$
d\left({ }^{i j j} \mathrm{O}_{3}\right)_{\text {tot }} / d t=\left(k_{j, i j}^{s}+k_{j, i j}^{a s}+k_{i, j j}^{a s} K_{j, i j}^{i, j j}\right){ }^{j} \mathrm{O} \cdot{ }^{i j} \mathrm{O}_{2} .
$$

For the formation of ${ }^{j j j} \mathrm{O}_{3}$ we have

$$
d^{j j j} \mathrm{O}_{3} / d t=\omega^{j j j} \mathrm{O}_{3}^{*}=k_{j, j j}^{s}{ }^{j} \mathrm{O} \cdot{ }^{j j} \mathrm{O}_{2} .
$$

Equations (4.6) and (4.7) yield a value for $\left({ }^{i j j} \mathrm{O}_{3}\right)_{\text {tot }}{ }^{j j j j} \mathrm{O}_{3}$ in terms of the ratio ${ }^{i j} \mathrm{O}_{2} /{ }^{j j} \mathrm{O}_{2}$. By analogy with the $\delta$ in general use for trace isotopes, given by Eq. (2.12), we may define one type of enrichment ${ }^{i} \delta_{j j}$ as the ${ }^{i} \mathrm{O} /{ }^{j} \mathrm{O}$ content in the ozone product divided by that in oxygen, minus 1 , that is

$$
{ }^{i} \delta_{j j}=\frac{\left({ }^{i j j} \mathrm{O}_{3}\right)_{\text {tot }}}{3^{j j j} \mathrm{O}_{3}} / \frac{{ }^{i j} \mathrm{O}_{2}}{2^{j j} \mathrm{O}_{2}}-1
$$

To calculate the denominator in such a quantity, it is necessary either to measure the concentrations of the isotopically different $\mathrm{O}_{2}$ 's or to use overall isotopic composition of the mixture and the equilibrium constants for equilibria among the various isotopically different $\mathrm{O}_{2}$ 's.

We then have

$$
{ }^{i} \delta_{j j}=\left[2\left(k_{j, i j}^{s}+k_{j, i j}^{a s}+k_{i, j j}^{a s} K_{j, i j}^{i, j j}\right) / 3 k_{j, j j}^{s}\right]-1 .
$$

Upon introducing Eq. (1.7) for the various recombination rate constants it follows that:

$$
{ }^{i} \delta_{j j}=\frac{2}{3} \frac{\sum \int\left(\zeta_{j i j} \rho_{j i j}+\zeta_{i j j} \rho_{i j j}\right) e^{-E / k_{B} T} d E / Q_{j, i j}}{\sum \int \zeta_{j j j} \rho_{j j j} e^{-E / k_{B} T} d E / Q_{j, j j}}-1 .
$$

We note from this equation for the scrambled mixture that, in contrast with Eq. (1.7) for the individual rate constants, the $Y^{\dagger}$ present in the latter has disappeared.

If we equate approximately the sum-integral over $\zeta_{j j j} \rho_{j j j} / 2 Q_{j, j j}$ to that over $\zeta_{j i j} \rho_{j i j} / Q_{j, i, j}$, the factor of 2 compensating for a difference in symmetry numbers of the $Q$ 's we would then have

$$
{ }^{i} \delta_{j j}=\frac{\sum \int\left(\zeta_{i j j} \rho_{i j j}-2 \zeta_{j i j} \rho_{j i j}\right) e^{-E / k_{B} T} d E / Q_{j, i j}}{3 \sum \int \zeta_{j j j} \rho_{j j j} e^{-E / k_{B} T} d E / Q_{j, j j}} .
$$

Equation (4.11) reduces, as it should, to Eq. (2.20) when $j$ $=6$ and $i=q$.

If we again introduce asymmetric and symmetric recombination rate constants $k_{a s}$ and $k_{s}$, as in Eqs. (2.22) and (2.23), we again obtain

$$
{ }^{i} \delta_{j j}=\frac{2}{3} \frac{k_{a s}-k_{s}}{k_{s}},
$$

whose counterpart in Sec. II is Eq. (2.24).

A definition which has been used for the enrichment for an ozone molecule of mass $M$ relative to ${ }^{48} \mathrm{O}_{3}$ in heavily enriched mixtures is $E^{15}$

$$
E=\left[\left(\frac{M_{\mathrm{O}_{3}}}{{ }^{48} \mathrm{O}_{3}}\right)_{\text {meas }} /\left(\frac{M_{\mathrm{O}_{3}}}{{ }^{48} \mathrm{O}_{3}}\right)_{\text {calc }}\right]-1,
$$

whose denominator is calculated statistically from the isotopic composition of the oxygen.

The ratio of formation of the ozones is, from Eq. (4.7) with $j=6$, and from Eq. (4.6)

$$
\frac{\left({ }^{i j j} \mathrm{O}_{3}\right)_{\mathrm{tot}}}{{ }^{666} \mathrm{O}_{3}}=\frac{{ }^{j} \mathrm{O} \cdot{ }^{i j} \mathrm{O}_{2}}{{ }^{6} \mathrm{O} \cdot{ }^{66} \mathrm{O}_{2}} \frac{k_{j, i j}^{s}+k_{j, i j}^{a s}+k_{i, j j}^{a s} K_{j, i j}^{i, j j}}{k_{6,66}^{s}} .
$$

If $X_{i}$ denotes the mole fraction of isotope $i$ present in the oxygen molecules, one type of statistically calculated concentration of the $i j j$ ozone, summed over both isotopomers and without regard to individual properties such as zeropoint energies of real molecules, is given by $3 \mathrm{O}_{3} X_{i} X_{j}^{2}$, where $\mathrm{O}_{3}$ is the total ozone concentration. Similarly, the calculated concentration of the sum of ijk ozones is $6 \mathrm{O}_{3} X_{i} X_{j} X_{k}$, and that of ${ }^{666} \mathrm{O}_{3}$ is $\mathrm{O}_{3} X_{6}^{3}$. The $\left(\left({ }^{i j j} \mathrm{O}_{3}\right)_{\text {tot }} /{ }^{666} \mathrm{O}_{3}\right)_{\text {calc }}$ ratio then equals $3 X_{i} X_{j}^{2} / X_{6}^{3}$.

We now have from Eq. (4.14)

$$
E_{i j j}=\frac{\left({ }^{j} \mathrm{O} / X_{j}\right)\left({ }^{i j} \mathrm{O}_{2} / X_{i} X_{j}\right)}{3\left({ }^{6} \mathrm{O} / X_{6}\right)\left({ }^{66} \mathrm{O}_{2} / X_{6}^{2}\right)} \frac{k_{j, i j}^{s}+k_{j, i j}^{a s}+k_{i, j j}^{a s} K_{j, i j}^{i, j j}}{k_{6,66}^{s}}-1 .
$$

We note that the ratio of atomic concentrations ${ }^{j} \mathrm{O} /{ }^{6} \mathrm{O}$ equals $\left({ }^{j j} \mathrm{O}_{2} /{ }^{6 j} \mathrm{O}_{2}\right) K_{6, j j}^{j, 6 j}$, and also note that

$$
Q_{i j}^{2} / 4 Q_{i i} Q_{j j} \simeq 1, \quad i \neq j .
$$

In fact, the actual ratios in Eq. (4.16) for $(i, j)=(16,17),(17$, $18)$, and $(16,18)$, are extremely close to unity, namely, $0.998,0.998$, and 0.996, respectively. One can then show that $^{73(a)}$

$$
{ }^{i j} \mathrm{O}_{2} \simeq 2 X_{i} X_{j} \mathrm{O}_{2}, \quad{ }^{i i} \mathrm{O}_{2} \simeq X_{i}^{2} \mathrm{O}_{2},
$$

where $\mathrm{O}_{2}$ is the total concentration of the oxygen molecules, regardless of isotope. One then obtains 


$$
\begin{aligned}
& E_{i j j}=\frac{\left(k_{j, i j}^{s}+k_{j, i j}^{a s}+k_{i, j j}^{a s} K_{j, i j}^{i, j j}\right) K_{6, j j}^{j, 6 j}}{3 k_{6,66}^{s}}-1, \quad j \neq 6, \\
& E_{i j j}=\frac{2\left(k_{j, i j}^{s}+k_{j, i j}^{a s}+k_{i, j j}^{a s} k_{j, i j}^{i, j j}\right)}{3 k_{6,66}^{s}}-1, \quad j=6 .
\end{aligned}
$$

The latter relation reduces to Eq. (2.13) for $\delta$, and so permits in that way a direct comparison of the two sets of results. ${ }^{73(b)}$

The completely symmetric isotopomer ${ }^{j j j} \mathrm{O}$ has an enrichment denoted by $E_{j j j}$

$$
E_{j j j}=\frac{{ }^{j j j} \mathrm{O}_{3} /{ }^{666} \mathrm{O}_{3}}{\left({ }^{j j j} \mathrm{O}_{3} /{ }^{666} \mathrm{O}_{3}\right)_{\text {calc }}}-1 .
$$

The ${ }^{j j j} \mathrm{O}_{3} /{ }^{666} \mathrm{O}_{3}$ ratio obtained from Eq. (4.7) is

$$
\frac{{ }^{j j j} \mathrm{O}_{3}}{{ }^{666} \mathrm{O}_{3}}=\frac{k_{j, j j}^{s}}{k_{6,66}^{s}} \frac{{ }^{j} \mathrm{O} \cdot{ }^{j j} \mathrm{O}_{2}}{{ }^{66} \mathrm{O}_{2}} .
$$

Following the prescription given earlier, the $\left({ }^{j j j} \mathrm{O}_{3} /{ }^{666} \mathrm{O}_{3}\right)_{\text {calc }}$ equals $\left(X_{j} / X_{6}\right)^{3}$. Once again, the ${ }^{j} \mathrm{O} /{ }^{6} \mathrm{O}$ is written as $\left({ }^{j j} \mathrm{O}_{2} /{ }^{6 j} \mathrm{O}_{2}\right) K_{6, j j}^{j, 6 j}$. Using Eq. (4.17) one finds

$$
E_{j j j}=\frac{k_{j, j j}^{s} K_{6, j j}^{j, 6 j}}{2 k_{6,66}^{s}}-1, \quad(j \neq 6),
$$

and is zero when $j=6$. Thus, even if $k_{j, j j}^{s}$ and $k_{6,66}^{s}$ were equal, because of partial cancellation in each of the $\zeta \rho / Q$ 's appearing in these rate constants, the $K_{6, j j}^{j, 6 j} / 2$, which is less than unity for $j=7$ and 8 , would make $E_{j j j}$ negative.

For calculating the enrichment $E_{i j k}$ of the completely asymmetric isotopomers we have for the rate of formation

$$
\begin{aligned}
d^{i j k} \mathrm{O}_{3} / d t & =\omega\left({ }^{i j k} \mathrm{O}_{3}^{*}\right)_{\text {tot }} \\
& =k_{i, j k}^{a s}{ }^{i} \mathrm{O} \cdot{ }^{j k} \mathrm{O}_{2}+k_{j, i k}^{a s}{ }^{j} \mathrm{O} \cdot{ }^{i k} \mathrm{O}_{2}+k_{k, i j}^{a s}{ }^{k} \mathrm{O} \cdot{ }^{i j} \mathrm{O}_{2} .
\end{aligned}
$$

If two equilibrium constants are introduced

$$
{ }^{i} \mathrm{O}+{ }^{j k} \mathrm{O}_{2} \rightleftharpoons{ }^{j} \mathrm{O}+{ }^{i k} \mathrm{O}_{2}, \quad K_{i, j k}^{j, i k}
$$

and

$$
{ }^{i} \mathrm{O}+{ }^{j k} \mathrm{O}_{2} \rightleftharpoons{ }^{k} \mathrm{O}+{ }^{i j} \mathrm{O}_{2}, \quad K_{i, j k}^{k, i j},
$$

we have

$$
\frac{{ }^{i j k} \mathrm{O}_{3}}{{ }^{666} \mathrm{O}_{3}}=\frac{\left(k_{i, j k}^{a s}+k_{j, i k}^{a s} K_{i, j k}^{j, i k}+k_{k, i j}^{a s} K_{i, j k}^{k, i j}\right)^{i} \mathrm{O} \cdot{ }^{j k} \mathrm{O}_{2}}{k_{6,66}^{s}{ }^{6} \mathrm{O} \cdot{ }^{66} \mathrm{O}_{2}} .
$$

To obtain $\left({ }^{i j k} \mathrm{O}_{3} /{ }^{666} \mathrm{O}_{3}\right)$ calc we use the earlier result for it, $6 X_{i} X_{j} X_{k} / X_{6}^{3}$, write ${ }^{i} \mathrm{O} /{ }^{6} \mathrm{O}$ in Eq. (4.25) as $\left({ }^{i i} \mathrm{O}_{2} /{ }^{6 i} \mathrm{O}_{2}\right) K_{6, i i}^{i, 6 i}$, and use Eq. (4.14) to obtain

$$
E_{i j k}=\frac{\left(k_{i, j k}^{a s}+k_{j, i k}^{a s} K_{i, j k}^{j, i k}+k_{k, i j}^{a s} K_{i, j k}^{k, i j}\right)}{3 k_{6,66}^{s}}-1, \quad(i=6) .
$$

We next consider for comparison an alternative definition of the calculated ratio, obtained, instead, by ignoring differences in rate constants leading to total product. From Eq. (4.14) we have

$$
\left(\frac{\left({ }^{i j j} \mathrm{O}_{3}\right)_{\text {tot }}}{{ }^{666} \mathrm{O}_{3}}\right)_{\text {calc }}=\frac{{ }^{j} \mathrm{O} \cdot{ }^{i j} \mathrm{O}_{2}+{ }^{i} \mathrm{O} \cdot{ }^{j j} \mathrm{O}_{2}}{{ }^{6} \mathrm{O} \cdot{ }^{66} \mathrm{O}_{2}} .
$$

The ratios ${ }^{j} \mathrm{O} /{ }^{6} \mathrm{O}$, and ${ }^{i} \mathrm{O} /{ }^{6} \mathrm{O}$ can be evaluated as before in terms of various ratios of the different ${ }^{i j} \mathrm{O}_{2}$ molecules, using known equilibrium constants for Eq. (4.3). With the definition in Eq. (4.27) we then have from Eqs. (4.14), (4.17), and (4.27) an enrichment denoted by $\bar{E}_{i j j}$

$$
\bar{E}_{i j j}=\frac{k_{j, i j}^{s}+k_{j, i j}^{a s}+k_{i, j j}^{a s} K_{j, i j}^{i, j j}}{k_{6,66}^{s}\left(1+K_{j, i j}^{i, j j}\right)}-1 .
$$

This definition differs from the $E_{i j j}$ in Eq. (4.18) by having the factor $1 /\left(1+K_{j, i j}^{i, j j}\right)$ instead of the $K_{6, j j}^{j, 6 j} / 3$. As a result of this difference, the expression for $\bar{E}_{i j j}$ proves to have a more symmetrical form than $E_{i j j}$, when the $K^{\prime}$ s are expressed in terms of partition functions.

We next consider $\bar{E}_{j i j}$. The ${ }^{j j j} \mathrm{O}_{3} /{ }^{666} \mathrm{O}_{3}$ ratio is obtained from Eq. (4.20) and $\left({ }^{j j j} \mathrm{O}_{3} /{ }^{666} \mathrm{O}_{3}\right)_{\text {calc }}$ is obtained from the latter by setting $k_{j, j j}^{s}=k_{6,66}^{s}$. We thus find

$$
\bar{E}_{j j j}=\frac{k_{j, j j}^{s}}{k_{6,66}^{s}}-1 .
$$

This $\bar{E}_{j j j}$ equals zero if $k_{j, j j}^{s}=k_{6,66}^{s}$.

We consider next $\bar{E}_{i j k}$ with $i, j$, and $k$ unequal. For the ratio $\left({ }^{i j k} \mathrm{O}_{3} /{ }^{666} \mathrm{O}_{3}\right)$ calc we equate all rate constants in Eq. (4.25), and so obtain

$$
\bar{E}_{i j k}=\frac{k_{i, j k}^{a s}+k_{j, i k}^{a s} K_{i, j k}^{j, i k}+k_{k, i j}^{a s} K_{i, j k}^{k, i j}}{k_{6,66}^{s}\left(1+K_{i, j k}^{j, i k}+K_{i, j k}^{k, i j}\right)}-1 .
$$

When the $K$ 's are expressed in terms of partition functions, Eq. (4.30) is seen to be symmetric in all three indices.

We return to the E's and introduce Eq. (1.7) for the $k$ 's and also express the equilibrium constants in Eq. (4.18) in terms of the partition functions in the center-of-mass system of coordinates. We find

$E_{i j j}=\frac{\sum \int\left(\zeta_{j i j} \rho_{j i j}+\zeta_{i j j} \rho_{i j j}\right) e^{-E / k_{B} T} d E /\left(Q_{j, i j} Q_{6, j j} / Q_{j, 6 j}\right)}{3 \sum \int \zeta_{666} \rho_{666} e^{-E / k_{B} T} d E / Q_{6,66}}$

$-1$.

This result can be rewritten approximately in the form (4.12) by writing $\Sigma \int \zeta_{j i j} \rho_{j i j} \exp \left(-E / k_{B} T\right) d E / Q_{j, i j}$ as $\frac{1}{2} k_{s}$ (since $\rho_{j i j}$, but not $Q_{i, j i}$, has a symmetry number of 2 ), write $\sum \int \zeta_{i j j} \rho_{i j j} \exp \left(-E / k_{B} T\right) d E / Q_{i, j j}$ as $2 k_{a s}$ and $\Sigma \int \zeta_{666} \rho_{666}$ $\times \exp \left(-E / k_{B} T\right) d E /\left(Q_{j, i j} Q_{6, j j} / Q_{6,6 j}\right)$ as $k_{s}$. One then obtains

$$
E_{i j j}=\frac{2}{3} \frac{k_{a s}-k_{s}}{k_{s}} \text {. }
$$

Because some of the above equalities are only approximations, deviations of $E_{i j j}$ must occur from this value.

For $E_{j j j}$ we obtain, similarly

$$
E_{j j j}=\frac{\sum \int \zeta_{j j j} \rho_{j j j} e^{-E / k_{B} T} d E / Q_{j, j j}\left(Q_{6, j j} / Q_{j, 6 j}\right)}{\sum \int \zeta_{666} \rho_{666} e^{-E / k_{B} T} d E / Q_{6,66}}-1 .
$$

As already noted, even if the $k_{j, j j}^{s}$ equaled the $k_{6,66}^{s}$, the $Q_{6, j j} / Q_{j, 6 j}$ would cause $E_{j j j}$ to be negative for $j=7$ and 8 .

The enrichment $E_{i j k}$ for the completely asymmetric isotopomers is obtained from Eqs. (4.26) and (1.7) 


$$
E_{i j k}=\frac{\sum \int\left(\zeta_{i j k} \rho_{i j k}+\zeta_{i k j} \rho_{i k j}+\zeta_{j i k} \rho_{j i k}\right) e^{-E / k_{B} T} d E /\left(Q_{i, j k} Q_{6, i i} / Q_{i, 6 i}\right)}{6 \sum \int \zeta_{666} \rho_{666} e^{-E / k_{B} T} d E / Q_{6,66}}-1 .
$$

If each term in the numerator of Eq. (4.34) were written approximately as $k_{a s}$, there being three $\zeta \rho$ terms, and if the sum-integral term in the denominator is written as $k_{s}$, we would have

$$
E_{i j k}=\frac{k_{a s}-k_{s}}{k_{s}},
$$

which may be compared with Eq. (4.32) for $E_{i j j}$.

Related expressions can be written for the $\bar{E}$ 's. For example, from the equations in this section one finds that 1 $+\bar{E}_{i j j}$ equals $\left(1+E_{i j j}\right) 3 Q_{6, j j} Q_{j, i j} /\left(Q_{j, i j}+Q_{i, j j}\right) Q_{j, 6 j}$, when $j \neq 6$, and equals $\left(1+E_{i j j}\right) 3 Q_{j, i j} / 2\left(Q_{j, i j}+Q_{i, j j}\right)$ when $j$ 6. Further, $1+\bar{E}_{i j k}$ equals $\left(1+E_{i j k}\right) 3 Q_{6, j k} /\left(Q_{6, j k}+Q_{j, 6 k}\right.$ $\left.+Q_{k, 6 j}\right)$ for $j, k \neq 6$, and $1+\bar{E}_{j j j}$ equals $\left(1+E_{j j j}\right) 2 Q_{6, j j} /$ $Q_{j, 6 j}$ when $j \neq 6$. When $j=6, \bar{E}_{j j j}=E_{j j j}=0$. We use these relations next.

As a guide to future theoretical calculations of the rate constants and of the enrichments, it is useful to see whether $E$ or $\bar{E}$ is more constant in value, both for the different $i j j$ molecules and for the jjj ones. For $(i, j)=(7,6),(6,7),(8,6)$, $(6,8),(8,7),(7,8)$ we have $E_{i j j}=0.11,0.12,0.13,0.14,0.095$, 0.083 , respectively, ${ }^{23}$ while calculated from these values using the expressions relating $1+\bar{E}$ to $1+E$ and various partition function ratios, one finds $\bar{E}_{i j j}=0.13,0.15$, $0.16,0.21,0.16,0.16$, respectively. Using the $E_{i j k}$ with $i, j, k$ unequal, ${ }^{23} 0.18$, the $\bar{E}_{i j k}$ calculated from it is 0.23 . Among the $\bar{E}_{i j j}$ 's the $(i, j)=(6,8)$ is anomalously high but the remainder are nearly equal in value.

If one assumed for the moment, that all $k_{j, j j}^{s}$ 's were equal (and they need not be), and so all $\bar{E}_{j j j}$ 's vanished, then the calculated values of $E_{j j j}$ for $j=7$ and 8 are -0.04 and -0.07 , while the experimental $E_{j j j}$ 's are -0.02 and $-0.05 .^{23}$

\section{INTRAMOLECULAR COUPLING, SYMMETRY, AND A SOURCE OF ADDITIONAL STATES}

In this section we consider possible sources for an extra density of reactive states for QOO as compared with the density for the symmetric molecules $\mathrm{OQO}$ and $\mathrm{O}_{3}$. Whereas $\mathrm{O}_{3}$ is only formed from a symmetric molecule $\mathrm{O}_{2}$ plus $\mathrm{O}$, the $\mathrm{OQO}$ is only formed from an asymmetric molecule QO plus $\mathrm{O}$. Thus, the similarity of behavior of $\mathrm{OQO}$ and $\mathrm{O}_{3}$ appears to be associated with the symmetry of these molecules themselves rather than with symmetry effects occurring during their formation from the reactants. This point suggests that we should focus for the present on the behavior of the vibrationally excited ozone as the source of the mass-independent isotope effect, rather than on an evolution along reaction coordinate, as a working hypothesis. The pragmatic test, of course, will be provided by how successfully the present mechanism explains the experimental facts and predict others.
A third possible source of the mass-independent isotope effect involves spin-orbit coupling to low-lying triplet states: At large $\mathrm{O}_{2}-\mathrm{O}$ separation distances some 27 spinorbit electronic states reach approximately the same asymptotic potential energy. Aside from the fact that the states may be energetically inaccessible to an ozone molecule in its electronic ground state, except perhaps at the large separation distances,${ }^{74-80}$ they do not, on examination, provide a symmetry effect over and above the usual symmetry number of two, and so do not account for a $\delta \neq 0$. This result, which has its origin in angular momentum conservation, is established later in Appendix B.

We come, instead, to a different effect of symmetry, namely, on possible deviations from RRKM ("statistical") behavior. Specifically, we consider the effect of symmetry on the density of internal resonances and the intramolecular coupling of the zeroth-order quantum states of the ozone. We consider first the dynamical behavior in classical terms.

Classically, a bound state of a molecule can display dynamically "quasi-periodic" behavior or chaotic behavior or some mixture, for example, islands of quasi-periodic behavior in phase space in an otherwise chaotic sea. Quasi-periodic motion has $N$ constants of the motion ( $N$ action variables) in a $2 N$ dimensional rovibrational phase space. ${ }^{57,81}$ Thus, the molecule moves on an $\mathrm{N}$-dimensional torus rather than throughout the $2 N$-dimensional space. We shall also use this label "torus" rather loosely to describe the unbound situation where in some limited region of phase space the torus is connected to a tube, an open exit channel. With some tori being connected to exit channels leading to reaction products, or some not at all connected, this torus effect leads to a state-specific unimolecular rate constant $k$, rather than to $k$ 's which depend only on the well-known constants of the motion, such as the angular momentum $J$ and the rovibrational energy $E$ and other, if any, conserved quantity $K$. In this case, the behavior is non-RRKM.

In the other limit, this quasi-periodic motion has given way to a chaotic behavior, the tori have disappeared, so that the system now occupies the $2 N$-dimensional phase space, limited only by the conditions of constant $E$, $J$ and of any other constants of the motion $K$. We note that in a rigid rotor-harmonic oscillator limit the $\mathrm{N}$-dimensional tori exist, and continue to do so, depending on the energy, even with the addition of small anharmonic and other coupling terms. While the tori persist at first with such added perturbations they begin to break down as the terms increase and various overlapping internal resonances then develop. The motion becomes increasingly chaotic with increasing energy or increasing coupling. Slater's theory of unimolecular reactions ${ }^{82}$ was based on a quasi-periodic model for the energized molecule in which the modes were also harmonic oscillators. Slater's calculated unimolecular rate constants then depended on the amplitudes of these oscillators, rather than only on $E$ and $J$. These amplitudes can be simply ex- 
pressed in terms of the $N$ constants of the motion, the action variables. (In the case of an unbound, i.e., dissociating, molecule one of these $N$ constants is the energy, and its conjugate variable is time.) (Use of the latter was made of this relation in a semiclassical treatment of collisions. ${ }^{83}$ )

For several reasons the tendency to chaotic motion, or to a quantum mechanical equivalent (which has been described elsewhere in terms of "overlapping resonances" or "overlapping avoided crossings"), ${ }^{57,84}$ can be expected to be greater for QOO than for $\mathrm{O}_{3}$ or OQO. We consider this aspect in terms of internal resonances and couplings among zeroth-order quantum states: We note first, however, that both in the classical regime and in a quantum equivalent of the classical quasi-periodic motion, some of the states at any total $E$ and $J$ may not be linked to an exit channel leading to products. The remaining reactive states, thereby, have a lower density of states than the total density of states. Thus, when the chaotic behavior is greater, this density of reactive states more nearly approaches the full density. The $\rho$ for $\mathrm{O}_{3}$ and OQO can be somewhat less, on this basis, than that of QOO, even apart from the symmetry factor of 2.

We examine now some intramolecular coupling terms, first for $C_{2 v}$ molecules, such as $\mathrm{O}_{3}$ and OQO, in the ${ }^{1} A_{1}$ ground electronic state. In the Hamiltonian, only intramolecular coupling terms which are of $A$ symmetry contribute. Many otherwise contributing terms are therefore now absent. Recalling that the $\mathrm{O}-\mathrm{O}$ symmetric stretch $\left(q_{1}\right)$ and the $\mathrm{OOO}$ bend $\left(q_{2}\right)$ in $\mathrm{O}_{3}$ have $A_{1}$ symmetry, while the $\mathrm{O}-\mathrm{O}$ asymmetric stretch $\left(q_{3}\right)$ has $B_{2}$ symmetry, anharmonic terms such as $q_{1} q_{2} q_{3}$ and $q_{1} q_{2} q_{3}^{3}$, are of $B$ symmetry and are, therefore, absent. ${ }^{85}$ Every vibrational state $\left(\mathrm{v}_{1}, \mathrm{v}_{2}, \mathrm{v}_{3}\right)$ is allowed, provided the rotational-nuclear spin state of the ${ }^{1} A_{1}$ state is such that the entire rovibrational-nuclear spin state is of $A$ symmetry when the $\mathrm{O}$ has an even number of nucleons or of $B$ symmetry when the $\mathrm{O}$ is ${ }^{17} \mathrm{O}$. Because of this requirement, half of the rotational states are absent in each case. For any zeroth-order vibrational state $\left(\mathrm{v}_{1}, \mathrm{v}_{2}, \mathrm{v}_{3}\right)$ some of the terms in the Hamiltonian which couple it to other states are, as seen above, absent. However, all such terms are present for the $C_{s}$ molecule QOO, although their coefficients may be small. Thus, one expects that QOO has a greater tendency to being more "chaotic" than OQO or $\mathrm{O}_{3}$, since for any vibrational state there are more intramolecular coupling terms, but the effect may be small, since the new coupling coefficients in the $C_{s}$ molecule are small. In fact, only a total of $10 \%$ is needed in the total increased density of states to explain the mass-independent results.

Related remarks apply for the Coriolis coupling terms $J_{i} p_{i}(i=a, b, c)$, which have vibrational and total angular momentum components $p_{i}$ and $J_{i}$, respectively. ${ }^{85}$ For a triatomic molecule only the component $p_{c}$ perpendicular to the plane of the three atoms is nonzero. For $\mathrm{O}_{3}$ or OQO, $J_{c}$ has $B_{2}$ symmetry, and so $p_{c}$ must also be of $B$ symmetry (e.g., $B_{2} \otimes B_{2}=A_{1}$ ). The $p_{c}$ contains only the asymmetric stretch $q_{3}$ and one of the $A_{1}$ vibrations, or more of them if the coefficient of $J_{c} p_{c}$ is expanded in the coordinates. For a $C_{s}$ molecule QOO, on the other hand, the only point symmetry operations are the identity and a reflection $\sigma_{a b}$ with respect to the molecular plane. All three vibrations have $A^{\prime}$ symme- try, as do $p_{c}$ and $J_{c}$. Thus, none of the above restrictions on the Hamiltonian perturbation terms apply and so there are more Coriolis coupling terms for the $C_{s}$ molecules than for the $C_{2 v}$ ones, for any given vibrational state $\left(\mathrm{v}_{1}, \mathrm{v}_{2}, \mathrm{v}_{3}\right)$. Similarly, there are more symmetry-allowed centrifugal distortion terms such as $q_{i} J_{\alpha} J_{\beta}$ and $q_{i} q_{j} J_{\alpha} J_{\beta}$ for QOO than for OQO or $\mathrm{O}_{3}$. Spectroscopic studies on selected isotopically different molecules, whether of ozone or of related molecules, can determine how significant these extra coupling terms are.

In summary, in addition to the usual symmetry number of a factor of 2, there are added restrictions on the various intramolecular coupling terms of the symmetric molecules, so that the number of coupling terms is somewhat less for $\mathrm{O}_{3}$ or OQO than for QOO. In the space of rovibrational states, there are also for any given $E$ and $J$ twice as many rovibrational states for $C_{s}$ molecules, and so there is a greater density of internal rovibrational resonances for them. One expects, thereby, for both reasons, a greater tendency to more chaotic behavior and so a higher density of reactive states in the $C_{s}$ molecules. Again, using various spectroscopic signatures one can attempt to determine how significant this difference of asymmetric and symmetric molecules is.

In Appendix B it was shown that angular momentum conservation prevented the electronic coupling of two electronic states from contributing to $\delta$. (Missing rovibrational states in the first electronic state, missing because of symmetry, lead to missing ones in the second. That is, the coupling affects the $\rho$ in the symmetric and the asymmetric molecules proportionally, and so does not contribute to $\delta$.) In the case of the properties of a single electronic state, on the other hand, certain anharmonic vibrational terms are absent for the symmetric molecule, regardless of the angular momentum $J$, and so the conservation of $J$ plays no role in this latter effect of coupling on $\delta$.

We have previously noted that increased pressure reduces the mass-independent isotope effect, ${ }^{16,20}$ eventually to zero. The reduction when the ambient gas was oxygen was similar to that when it was $80 \%$ nitrogen-20\% oxygen at the same total pressure, so it is not due to some additional reaction with $\mathrm{O}_{2}$. (The range of pressures studied in Ref. 20 was smaller than than in Ref. 16, however.) One possible explanation of the reduction in $\delta$ with increasing pressure, and it is just a possibility, is that the collisions can induce an intramolecular redistribution of quantum states, ${ }^{86}$ and so induce transitions between "reactive" and "nonreactive" quantum states. A second possibility, to be discussed elsewhere, is that some differences in the $k$ 's in Eq. (2.13) are due to differences in the low-energy regime and so are more sensitive to collisions (longer lifetimes).

\section{DISCUSSION}

We have mentioned a variety of experiments: (1) The similarity in enrichment of ${ }^{17} \mathrm{O}$ and ${ }^{18} \mathrm{O}$ in the recombination to form ozone, (2) the similarity of behavior for recombination, regardless of whether trace or heavily enriched isotopes are used, (3) the relation between the ratio of isotopomers QOO/OQO and the enrichment $\delta$, (4) the fact that enrichment occurs both for recombination to form ozone and for 
thermal dissociation of ozone, (5) the effect of pressure (or surfaces) on the enrichment, an effect which disappears at pressures far below the unimolecular fall-off transition region for ozone, and (6) the apparent dichotomy between observations of the mass-independent effect in trace systems or scrambled mixtures of heavily enriched isotopes on the one hand and the mass-dependent effect found in systematic studies of selected series of unscrambled heavily enriched systems on the other. We consider these results in this section.

In Sec. $\mathrm{V}$ a possible model was noted involving low lying electronic states (low lying at large values of the asymmetric stretching vibration $q_{3}$ ). We have shown in Appendix B why it does not add to the enrichment, though it can add to the density of reactive states for these molecules, and so do not consider it further.

The similarity in behavior of the different isotopes, which depends in scrambled mixtures only on the symmetry of the ozone molecule and not on the nuclear spins per se, is seen both in the trace experiments and in the heavily enriched ones. In the present treatment, the small nuclear hyperfine terms in the Hamiltonian were neglected, but symmetry effects were included: For every vibrational state of a symmetrical $C_{2 v}$ molecule half the rotational states are missing. Which half remains depends only on the symmetry of the nuclear spin-vibrational wave function for this ${ }^{1} A_{1}$ electronic ground state. Because rotational states are closely spaced, this difference for the different isotopes is insignificant.

In experimental studies of heavily enriched oxygen isotopes in scrambled mixtures there is strong evidence of a symmetry effect: The enhancement of the 678 isotopic ozone is roughly $\frac{3}{2}$ that of the average of the many ijj ozones $(j$ $\neq i$ ), consistent with Eqs. (4.18) and (4.28), while the 666, 777 , and 888 isotopomers show a small mass-dependent depletion effect. ${ }^{23}$ Further, according to equations such as Eqs. (2.24) and (4.12) the enrichment effect for systems with trace Q and for systems with heavily enriched Q should be similar, a result also consistent with existing experiments. We compared in Sec. IV the values of the $E$ 's and $\bar{E}$ 's for the heavily enriched mixtures, to see which was more constant. We have seen that except for one anomalous result (the value of 0.21 for $\bar{E}_{688}$ ) the other $\bar{E}_{i j j}$ 's were about equal, 0.130.16 . We return later to the contrasting studies on unscrambled mixtures of the heavily enriched systems, the apparent dichotomy that results, and a possible explanation.

We discuss next the relation in Eq. (2.14) between the ratio of the isotopomers, $\mathrm{QOO} / \mathrm{OQO}$, for recombination and the enrichment factor, $\delta$ and namely $R=3 \delta$ when $k_{6,66}$ $\simeq 2 k_{6, q 6}^{s}$, as in Eq. (2.14). Experimentally, $\delta$ has been measured using the $\left({ }^{16} \mathrm{O},{ }^{18} \mathrm{O}\right)$ pair to be about $0.09,{ }^{16,20}$ so that the expected value for $R$ is about 0.27 . There is some experimental uncertainty in $R$, but depending on how the data are processed the mean value of $R$ is 0.19 or $0.27 .{ }^{17-19}$ The ratio $k_{6,66} / 2 k_{6, q 6}^{s}$ would need to be measured to adequately test Eq. (2.14).

We consider next the thermal dissociation. Initially it might seem surprising that an enrichment of the heavier isotopes occurs not only for the forward direction, where ozone is formed thermally from oxygen atoms and molecules, but also in the reverse reaction, the thermal dissociation of ozone. ${ }^{33-35}$ This paradox is resolved, we have seen, when it is realized that it is not the difference in properties of the reactants versus products, e.g., in the separated atom and molecule versus the ozone molecule, which is playing the major role in $\delta$, but rather the density of states of the vibrationally excited ozone. Specifically, the recombination and dissociation rate constants are given by Eqs. (1.7) and (1.8) in the low-pressure region.

The predicted enrichment $\delta$ of the oxygen formed from ozone dissociation, given by Eq. (3.12), is similar to that predicted for ozone formation by Eq. (2.20), when the $3 K / 2, K$ given by Eq. (3.13), is close to unity. Experimentally, however, the enrichment of the oxygen formed in the ozone dissociation is substantially less than that for the ozone formed from the oxygen. ${ }^{33-35}$ The observed enrichment $\delta$ for the ozone dissociation does increase with increasing temperature, a result which was attributed to a decreasing role of the surface, in the ozone decomposition, at the shorter reaction times prevailing at the higher temperatures. ${ }^{35}$ If the surface is indeed the main reason for the smaller $\delta$ values occurring in the ozone dissociation, this idea could be tested by studies at lower surface to volume ratios.

We consider next the possible explanation of the enrichment in terms of low-lying electronic states, and focus on coupling via spin-orbit terms, although other spin and/or rovibronic couplings can also occur. There are several lowlying triplet states, barely thermally accessible, ${ }^{74-80}$ except at large values of the asymmetric stretching coordinate $q_{3}$, where some 27 electronic states approach each other in energy. These states could increase the density of reactive states. For example, if the triplet states have longer range interaction than the ground singlet state, they could contribute via spin-orbit coupling to the density of states at large values of $\pm q_{3}$. However, it is shown in Appendix B that they make no additional contributions to the enrichment $\delta$, because of angular momentum conservation. A similar argument shows that any low-lying excited singlet state would not, by nonadiabatic coupling, contribute to the enrichment $\delta$ but could in principle add to the density of reactive states.

Systematic studies of various unscrambled, rather than scrambled mixtures, namely, studies of the ratio of recombination rates of ${ }^{i} \mathrm{O}+{ }^{j} \mathrm{O}{ }^{j} \mathrm{O}$ and ${ }^{i} \mathrm{O}+{ }^{i} \mathrm{O}{ }^{i} \mathrm{O}$, where $i$ and $j$ are 16,17 , or 18 , showed a clear mass-dependent trend: ${ }^{21,22}$ For reactions involving a light isotope $i$ in the atom and a heavy isotope $j$ in the molecule, the ratio $k_{i, j j}^{a s} / k_{j, j j}^{s}$. was clearly greater than unity, while in the opposite case $\left({ }^{i} \mathrm{O}\right.$ heavy and ${ }^{j j} \mathrm{O}_{2}$ light) the ratio of rate constants was near or less than unity. That there should be a qualitative difference in behavior in the two cases is evident from Eq. (1.7), when one examines the partitioning factor $N_{a}^{\dagger} /\left(N_{a}^{\dagger}+N_{b}^{\dagger}\right)$ and its dependence on differences in zero-point energies of the two transition states. Quantitative calculations, however, will be needed to assess more fully the appropriateness of this explanation and are being made. In contrast, we have seen in Sec. IV the reaction rates of the scrambled heavily enriched or trace systems no longer contain the partitioning factor. Again, theoretical calculations are needed to assess quantita- 
tively this role of the partitioning factor as an explanation of the difference between the scrambled ${ }^{23}$ and unscrambled ${ }^{21,22}$ mixtures. They have been made and will be presented elsewhere.

We consider further the behavior of a system where some of the quasi-periodic motion-based tori (or remnants thereof, when some chaos occurs) are not connected to the exit channel. In this case, the effective density of reactive states is reduced, namely, by the density of nonreactive states. Thereby, the rate of unimolecular dissociation of a molecule of any given energy $\left[N^{\dagger} / h \rho\right.$ in the standard notation in Eq. (1.2)] is correspondingly higher. ${ }^{87}$ It is interesting to compare this behavior with recently studied fluctuations from RRKM behavior, such as in the dissociation of formaldehyde, where the excess energy was produced by optical excitation. ${ }^{88}$ Here, the quantum state-dependent rate constants for the dissociation were distributed about the RRKM value, as a function of energy. Had some of the states been nonreactive, the remaining ones, would instead, have been consistently above the RRKM value. ${ }^{88,89}$

Among theories of the source of $\delta$ it was initially supposed $^{29,32,50}$ that a difference in symmetry numbers, a factor of 2, of QOO and $\mathrm{OOO}$ was the source of $\delta$. It was subsequently shown ${ }^{90}$ that this difference could not be the source. Indeed, as we have noted earlier, this difference in symmetry number is tacitly included in the definition of $\delta$.

Various other sources of $\delta$ have been proposed, but the one that is closest to the present one is that of Bates. ${ }^{91} \mathrm{He}$ postulated that in a reaction $\mathrm{Q}+\mathrm{O}_{2} \rightarrow \mathrm{QOO}^{*}$, there would initially be formed a molecule containing much of its excess energy in the newly formed QO bond, which was denoted by $\mathrm{Q} \sim \mathrm{OO}^{*}$. It would be different from $\mathrm{QO} \sim \mathrm{O}^{*}$, the $\mathrm{QOO} *$ initially formed from $\mathrm{QO}+\mathrm{O}$. There would be some equilibration time between these two forms of QOO*, but before that equilibration there are two species which should be counted separately, presumably, thus increasing the effective density of states of QOO* and so increasing, by deactivation, the QOO formed. There is, however, a major problem, even aside from the fact that for a nonlinear ozone $\mathrm{O} \sim \mathrm{OO}^{*}$ and $\mathrm{OO} \sim \mathrm{O}^{*}$ would also be distinct species and so no longer have a symmetry number of two. The main problem is that each species $\mathrm{Q} \sim \mathrm{OO} *$ and $\mathrm{QO} \sim \mathrm{O}^{*}$ would have only half the density of states $\rho$ as QOO* and so in RRKM theory would decompose twice as fast, thereby negating the advantage of having two distinguishable species. Quantitatively, this argument can be expressed using equations where the isotopic exchange reactions are dominant and set up an equilibrium between $\mathrm{Q}+\mathrm{O}_{2}$ and $\mathrm{QO}+\mathrm{O}$. A steady-state treatment then yields the desired proof.

We turn next to the transition state and, by unimolecular arguments, to an expectation for its behavior at low pressures, the region of particular interest for study of the massindependent isotope effect. Normally, this information on the transition state is obtained from the limiting bimolecular association rate at high pressures or from the pre-exponential factor in the unimolecular rate constant at those pressures. However, the high-pressure behavior of ozone formation or dissociation appears to be somewhat complicated, rather than being a simple limiting behavior. ${ }^{51}$ Thus, the desired infor- mation on the transition state should also be sought from another source.

Some insight into the nature of the transition state at high pressures, without the complications which occur in the actual system under those conditions, is obtained by measuring the exchange rate constant at low pressures: About half of the reactive collisions in Eq. (2.7) lead statistically, when $\omega \simeq 0$, to exchange. At high pressures, all of these collisions would lead, instead, to recombination, because of complete collisional deactivation of each vibrationally excited ozone molecule that is formed. Thus, information about the high pressure rate constant $k_{\text {rec }}^{a s}(\omega \rightarrow \infty)$ becomes available from $k_{\mathrm{ex}}$, even though $k_{\mathrm{ex}}$ is measured at low pressures, namely, the $k_{\text {rec }}^{a s}(\omega \rightarrow \infty)$ approximately equals $2 k_{\mathrm{ex}}$. A mathematical proof of this statement is obtained by comparing Eqs. (1.10) and (1.12) for $k_{\text {rec }}^{a s}(\omega \rightarrow \infty)$ and $k_{\mathrm{ex}}^{a}(\omega \rightarrow 0)$, respectively: We see that when $Y_{b}^{\dagger} \simeq \frac{1}{2}$, it follows that:

$$
k_{\mathrm{rec}}^{a s}(\omega \rightarrow \infty) \simeq 2 k_{\mathrm{ex}}^{a}(\omega \rightarrow 0),
$$

so establishing the above result. We shall explore the consequences of this result elsewhere. However, we note here that at $300 \mathrm{~K}$ the data on $k_{\text {rec }}$ in Ref. 51 clearly have not reached an upper limit as the pressure is increased, and so Eq. (6.1) provides a way of estimating $k_{\mathrm{rec}}^{a s}(\omega \rightarrow \infty)$ in this case. The $k_{\text {ex }}^{a}(\omega \rightarrow 0)$ in Eq. (6.1) is free from the complications occurring in the very high pressure experiments for $k_{\text {rec }}^{a s}$.

One caveat, however, remote perhaps, on this use of the isotopic exchange rate constant $k_{\text {ex }}$ to estimate accurately the high-pressure rate constant for ozone formation should be mentioned: If most of exchange in $\mathrm{Q}+\mathrm{O}_{2} \rightarrow \mathrm{QO}+\mathrm{O}$ had occurred via a nonrandomized molecule QOO*, in which the energy is almost entirely in the $q_{3}$ mode, instead of via a randomized $\mathrm{QOO}^{*}$, the estimated high-pressure rate constant $k_{\mathrm{rec}}^{a}$ would have been approximately equal to the exchange rate constant instead of twice that value.

Experimentally, it would be useful to have more information on the unusual recombination rate of the ${ }^{16} \mathrm{O}$ system, $\mathrm{O}+\mathrm{O}_{2} \rightarrow \mathrm{O}_{3}$ and the dissociation rate $\mathrm{O}_{3} \rightarrow \mathrm{O}+\mathrm{O}_{2}$ itself. At present there are steady-state experiments. ${ }^{51}$ While they are very helpful indeed, a direct time-resolved measurement of the dissociation rate constant as a function of energy would provide additional information, free from the uncertainties due to uncertain or unknown collisional deactivation rates. Experimentally, in systems such as $\mathrm{NCNO} \rightarrow \mathrm{NC}+\mathrm{NO}^{92}$ and $\mathrm{CH}_{2} \mathrm{CO} \rightarrow \mathrm{CH}_{2}+\mathrm{CO},{ }^{93}$ time-resolved picosecond studies as a function of internal energy have been made by photoexcitation to an electronically excited state which underwent a radiationless transition to a vibrationally excited ground electronic state. The transition occurred readily since there was a curve crossing below the dissociation energy threshold of the ground state. The situation in ozone is less favorable and it may be necessary to attempt double resonance pumpdump experiments to produce the ozone in its ground electronic state but with enough vibrational energy to allow dissociation. At present, the information of the lifetime of vibrationally excited ozone molecules with respect to dissociation in steady-state experiments is inferred only indirectly, namely from its competition with deactivating collisions. There is, however, uncertainty in the efficiency and detailed 
nature of the deactivating collisions. Direct measurement of lifetimes and their energy dependence would provide added insight into the extent to which dissociating vibrationally excited ozone behaves statistically or is non-RRKM. It may be stressed that the observed effect is small in absolute terms but profound in its effect on the slope, namely on $\delta$ or $E$.

It would also be useful to explore the difference in couplings, as reflected in spectra, of vibrationally excited asymmetric and symmetric molecules, such as $\mathrm{O}_{3}, \mathrm{SO}_{2}$, and $\mathrm{NO}_{2}$, where the O's are the same or are different $\mathrm{O}$ isotopes. Such information can provide additional insights into the dynamical behavior of these molecules.

The explanation discussed in the present article on the mass-independent isotope effect is, we believe, at least consistent with many experimental results on these ozone systems. It also suggests other experiments, as well as numerical calculations, which may provide added insight into topics such as RRKM versus non-RRKM effects in small molecules, and which can test further some of the ideas described. It will be interesting to see, in studying other recombinations, both neutral-neutral and ion-molecule, whether the smaller the binding energy the larger the effect, other things being equal. In particular, it will be interesting to see if it contributes to the very large isotopic effect reported ${ }^{41}$ for the $\mathrm{O}_{2}+\mathrm{O}_{2}^{+} \rightarrow \mathrm{O}_{4}^{+}$recombination reaction. In small systems with relatively low binding energy there may be fewer overlapping internal resonances and hence less statistical behavior. One interesting aspect about the mass-independent isotope effect, if the present explanation is correct, is how a small absolute effect (here 10\%) can be magnified dramatically, by yielding a factor of 2 in a slope, $\delta$.

\section{ACKNOWLEDGMENTS}

It is a pleasure to acknowledge the support of this research by the National Science Foundation and very helpful discussions with Dr. Yuri Georgievskii. We should also like to thank Professor Stuart Anderson for a helpful discussion on the experiments.

\section{APPENDIX A: RATE CONSTANTS FOR TWO CHANNELS}

We consider the rate of probability of formation of the energetic molecules in the energy interval $(E, E+d E)$ by recombination of the two reactants. The system moves along the reaction coordinate $q$ across the transition state hypersurface and is in some quantum state of the other coordinates (other than $q$ ). This probability rate is $(\dot{q} d p / h) \exp (-E /$ $\left.k_{B} T\right) / Q^{(1,2)}$, using standard arguments and notation. We note that $\dot{q} d p=d E$. For a given $E J K$ there are $N_{a}^{\dagger}$ such quantum states accessible in the entry channel $a$. The probability that such molecules, once formed, will be deactivated to form the ozone rather than disappear by re-dissociation is $\omega /[\omega$ $\left.+\left(N_{a}^{\dagger}+N_{b}^{\dagger}\right) / h \rho\right], \quad\left(N_{a}^{\dagger}+N_{b}^{\dagger}\right) / h \rho$ being the RRKM total unimolecular dissociation rate constant for the given (EJK) into both exit channels $a$ and $b$. The net rate of recombination is obtained by integrating over all energies and summing over all $J$ and $K$. One thus obtains Eq. (1.5) of the text. The equation for unimolecular dissociation into the $a$ exit channel, Eq. (1.6), is obtained by an analogous argument.

We consider next the derivation of Eq. (1.11) for the isotopic exchange rate constant $k_{\mathrm{ex}}^{a}$. In this case, following the argument given above, the probability that the energetic molecules, once formed, will dissociate into product channel $b$, rather than be deactivated or disappear by redissociation, is $\left(N_{b}^{\dagger} / h \rho\right) /\left[\omega+\left(N_{a}^{\dagger}+N_{b}^{\dagger}\right) / h \rho\right]$. The net isotopic exchange rate is again obtained by integrating over all energies and summing over all $J$ and $K$, thus yielding Eq. (1.11) of the text.

\section{APPENDIX B: EFFECT OF COUPLING ON $\rho$ AND LACK OF EFFECT ON $\delta$}

We first note that ozone is a near-prolate symmetric top, since its rotational constants $A, B$, and $C$ are $3.55,0.45$, and $0.39 \mathrm{~cm}^{-1} .^{70}$ For simplicity of notation, though not essential for the final conclusion, we treat in this appendix the ozone as a symmetric top. (The asymmetric top can be treated by adapting the results in Ref. 94.) By way of summary we show that while spin-orbit coupling of the ground electronic state to other electronic states can increase the density of reactive states it does not affect the enrichment $\delta$. The same argument applies, as we note below, for couplings to other electronic states.

We first recall that the spin-orbit matrix element coupling two electronic states can be written as ${ }^{95,96}$ $\left\langle\alpha^{\prime} \beta^{\prime} S^{\prime} J^{\prime} N^{\prime} K^{\prime}\left|H_{\mathrm{so}}\right| \alpha \beta S J N K\right\rangle$, where $|S J N K\rangle$ denotes an eigenvector having a spin quantum number $S$, total angular momentum quantum number $J$, rotational angular momentum quantum number $N$ and component $K$ of $N$ along the molecular top (or near-top) axis. The $\beta$ in the $|\alpha \beta\rangle$ eigenvector denotes the electronic orbital quantum number and $\alpha$ the remaining quantum numbers (vibrational, nuclear spin). The spin-orbit term $H_{\text {so }}$ in the Hamiltonian will be written for our purpose as $c \mathbf{L}_{\text {eff }} \cdot \mathbf{S}_{\text {eff }}$, where $c$ is a constant, $\mathbf{S}_{\text {eff }}$ is a difference of spin operators of the two electrons, and $\mathbf{L}_{\text {eff }}$ a difference, in effect, of their orbital angular momentum operators in this singlet-triplet calculation. (Only the difference terms contribute to the singlet-triplet matrix element.) Upon noting that the rotational angular momentum $\mathbf{N}=-\mathbf{S}+\mathbf{J}$, the eigenvector $|S J N K\rangle$ can be written in terms of body-fixed components of $-\mathbf{S}$ and $\mathbf{J}$, with quantum numbers denoted by $-S_{z}$ and $P$, respectively. The transformation of eigenvectors is given by ${ }^{96}$

$$
|S J N K\rangle=\sum_{-S_{z} P}\left|S J\left(-S_{z}\right) P\right\rangle\left\langle S J\left(-S_{z}\right) P \mid S J N K\right\rangle,
$$

where the coefficients of the new eigenvectors are the relevant Clebsch-Gordan coefficients. These new eigenvectors are convenient since $\mathbf{L}_{\text {eff }}$ is best represented as body-fixed in these Hund's case (b) molecules and so $\mathbf{S}_{\text {eff }}$ is then similarly represented.

In the case of the singlet state, which we will denote by the primes, $S_{z}^{\prime}=0, S^{\prime}=0$, so $J^{\prime}=N^{\prime}$ and $P^{\prime}=K^{\prime}$. That is, there is only one term in the sum (B1) for that electronic state. Thus, the $H_{\text {so }}$ matrix element becomes 


$$
\begin{aligned}
& \left\langle\alpha^{\prime} \beta^{\prime} S^{\prime} J^{\prime} N^{\prime} K^{\prime}\left|H_{\mathrm{so}}\right| \alpha \beta S J N K\right\rangle \\
& =c \sum_{-S_{z} P}\left\langle\alpha^{\prime} \mid \alpha\right\rangle\left\langle\beta^{\prime}\left|\mathbf{L}_{\mathrm{eff}}\right| \beta\right\rangle \cdot\left\langle 00\left|\mathbf{S}_{\mathrm{eff}}\right| S\left(-S_{z}\right)\right\rangle \\
& \quad \times\left\langle S J\left(-S_{z}\right) P \mid S J N K\right\rangle\left\langle J^{\prime} P^{\prime} \mid J P\right\rangle .
\end{aligned}
$$

The orbital symmetry of the electronic states determines which component or components of $\mathbf{L}_{\text {eff }}$ contribute and, since $\mathbf{L}_{\text {eff }} \cdot \mathbf{S}_{\text {eff }}$ is a scalar, it thereby determines which components of $\mathbf{S}_{\text {eff }}$ contribute. Since not only OQO and OOO are $C_{2 \mathrm{v}}$ molecules in terms of the electronic coordinates, but so is QOO, the $\mathbf{L}_{\text {eff }}, \mathbf{S}_{\text {eff }}$ pair of matrix elements in Eq. (B2) is the same for all three molecules.

The symmetry of the nuclear spin/vibrational quantum state $\left|\alpha^{\prime}\right\rangle$ will determine, together with the number, even or odd, of nucleons in the end O's in a symmetric molecule, which $\left|N^{\prime} K^{\prime}\right\rangle$ states are allowed for the ${ }^{1} A_{1}$ ground singlet state. ${ }^{85}$ Since $\left\langle J^{\prime} P^{\prime} \mid J P\right\rangle=\delta_{J J}^{\prime} \delta_{P P}^{\prime}$, and since $J^{\prime}=N^{\prime}$ and $P^{\prime}=K^{\prime}$ (singlet state) it follows that this condition of angular momentum conservation, represented by this expression for $\left\langle J^{\prime} P^{\prime} \mid J P\right\rangle$, imposes on the triplet state (the primed state) the same "allowedness," i.e., $J=N^{\prime}, P=P^{\prime}=K^{\prime}$. Half the $K^{\prime}$ states were missing in the singlet state (which half depends on the vibrational-electronic-nuclear spin state in that electronic state), and so half of the $P$ states of the triplet are also missing.

We see that this spin-orbit coupling, which can add to the density of reactive states, cannot thereby contribute differently to the number of states of ${ }^{i} \mathrm{O}^{i} \mathrm{O}^{i} \mathrm{O},{ }^{i} \mathrm{O}^{j} \mathrm{O}^{j} \mathrm{O}$, and ${ }^{i} \mathrm{O}^{j} \mathrm{O}^{i} \mathrm{O}$ molecules $(j \neq i)$ and so cannot contribute to the enrichment $\delta$. The same remarks apply to other couplings, which would replace the $H_{\text {so }}$. The condition $\left\langle J^{\prime} P^{\prime} \mid J P\right\rangle$ $=\delta_{J J^{\prime}} \delta_{P P^{\prime}}$ still prevails, with the same consequences on the "allowedness" of which states in the excited electronic state can be so coupled, and hence, on the enrichment factor $\delta$.

\footnotetext{
${ }^{1}$ R. N. Clayton, L. Grossman, and T. K. Mayeda, Science 182, 485 (1973).

${ }^{2}$ M. H. Thiemens and J. E. Heidenreich III, Science 219, 1073 (1983).

${ }^{3}$ K. Mauersberger, Geophys. Res. Lett. 8, 935 (1981).

${ }^{4}$ C. P. Rinsland, V. M. Devi, J.-M. Flaud, C. Camy-Peyret, M. A. H. Smith, and G. M. Stokes, J. Geophys. Res. 90, 10719 (1985); M. M. Abbas, J. Guo, B. Carli, F. Mencaraglia, M. Carlotti, and I. G. Nolt, ibid. 92, 13231 (1987).

${ }^{5}$ K. Mauersberger, Geophys. Res. Lett. 14, 80 (1987).

${ }^{6}$ B. Carli and J. H. Park, J. Geophys. Res. 93, 3851 (1988).

${ }^{7}$ A. Goldman, F. J. Murcray, D. G. Murcray, J. J. Kosters, C. P. Rinsland, J.-M. Flaud, C. Camy-Peyret, and A. Barbe, J. Geophys. Res. 94, 8467 (1989).

${ }^{8}$ B. Schueler, J. Morton, and K. Mauersberger, Geophys. Res. Lett. 17, 1295 (1990).

${ }^{9}$ D. Krankowsky, F. Bartecki, G. G. Klees, K. Mauersberger, and K. Schellenback, Geophys. Res. Lett. 22, 1713 (1995).

${ }^{10}$ F. W. Irion, M. R. Gunson, C. P. Rinsland, Y. L. Yung, M. C. Abrams, A. Y. Chang, and A. Goldman, Geophys. Res. Lett. 23, 2377 (1996).

${ }^{11}$ A. Meier and J. Notholt, Geophys. Res. Lett. 23, 551 (1996).

${ }^{12}$ J. C. Johnston and M. H. Thiemens, J. Geophys. Res. 102, 25395 (1997); see also Ref. 28.

${ }^{13}$ M. H. Thiemens and T. Jackson, Geophys. Res. Lett. 15, 639 (1988).

${ }^{14}$ M. H. Thiemens and T. Jackson, Geophys. Res. Lett. 14, 624 (1987).

${ }^{15}$ J. Morton, B. Schueler, and K. Mauersberger, Chem. Phys. Lett. 154, 143 (1989).

${ }^{16}$ M. H. Thiemens and T. Jackson, Geophys. Res. Lett. 17, 717 (1990).

${ }^{17}$ S. M. Anderson, J. Morton, and K. Mauersberger, Chem. Phys. Lett. 156, 175 (1989).
}

${ }^{18}$ N. W. Larsen, T. Pedersen, and J. Sehested, Int. J. Chem. Kinet. 23, 331 (1991).

${ }^{19}$ N. W. Larsen, T. Pedersen, and J. Sehested, ACS Symp. Ser. 502, 167 (1992).

${ }^{20}$ J. Morton, J. Barnes, B. Schueler, and K. Mauersberger, J. Geophys. Res. 95, 901 (1990).

${ }^{21}$ S. M. Anderson, D. Hülsebusch, and K. Mauersberger, J. Chem. Phys. 107, 5385 (1998).

${ }^{22}$ K. Mauersberger, B. Erbacher, D. Krankowsky, J. Günther, and R. Nickel, Science 283, 370 (1999).

${ }^{23}$ K. Mauersberger, J. Morton, B. Schueler, and J. Stehr, Geophys. Res. Lett. 20, 1031 (1993).

${ }^{24}$ M. H. Thiemens, ACS Symp. Ser. 502, 138 (1992).

${ }^{25}$ J. Sehested, O. J. Nielsen, H. Egsgaard, N. W. Larsen, T. Pedersen, L. K. Christensen, and M. R. Wiegell, J. Geophys. Res. 100, 20979 (1995).

${ }^{26}$ L. K. Christensen, N. W. Larsen, F. M. Nicolaisen, T. Pedersen, G. O. Sorensen, and H. Egsgaard, J. Mol. Spectrosc. 175, 220 (1996).

${ }^{27}$ J. Sehested, O. J. Nielsen, H. Egsgaard, N. W. Larsen, T. S. Andersen, and T. Pedersen, J. Geophys. Res. 103, 3545 (1998).

${ }^{28}$ M. H. Thiemens, Science 283, 341 (1999).

${ }^{29}$ J. E. Heidenreich III and M. H. Thiemens, J. Chem. Phys. 84, 2129 (1986).

${ }^{30}$ S. K. Bains-Sahota and M. H. Thiemens, J. Phys. Chem. 91, 4370 (1987).

${ }^{31}$ J. Yang and S. Epstein, Geochim. Cosmochim. Acta 51, 2011 (1987).

${ }^{32}$ J. Yang and S. Epstein, Geochim. Cosmochim. Acta 51, 2019 (1987).

${ }^{33}$ S. K. Bhattacharya and M. H. Thiemens, Geophys. Res. Lett. 15, 9 (1988).

${ }^{34}$ J. Wen and M. H. Thiemens, Chem. Phys. Lett. 172, 416 (1990).

${ }^{35}$ J. Wen and M. H. Thiemens, J. Geophys. Res. 96, 10911 (1991).

${ }^{36}$ J. E. Heidenreich III and M. H. Thiemens, Geochim. Cosmochim. Acta 49, 1303 (1985).

${ }^{37}$ J. Wen and M. H. Thiemens, Geophys. Res. Lett. 98, 12801 (1993).

${ }^{38}$ S. K. Bhattacharya and M. H. Thiemens, Z. Naturforsch. 44, 435 (1989).

${ }^{39}$ J. J. Colman, X. Xu, M. H. Thiemens, and W. C. Trogler, Science 273, 774 (1996).

${ }^{40}$ K. S. Griffith and G. I. Gellene, J. Chem. Phys. 96, 4403 (1992).

${ }^{41}$ K. S. Griffith and G. I. Gellene, ACS Symp. Ser. 502, 210 (1992).

${ }^{42}$ G. I. Gellene, J. Phys. Chem. 97, 34 (1993).

${ }^{43}$ R. K. Yoo and G. I. Gellene, J. Chem. Phys. 102, 3227 (1995).

${ }^{44}$ H. Craig, Geochim. Cosmochim. Acta 12, 133 (1957).

${ }^{45}$ J. Bigeleisen and M. Wolfsberg, Adv. Chem. Phys. 1, 16 (1958).

${ }^{46}$ Y. Matsuhisa, J. R. Goldsmith, and R. N. Clayton, Geochim. Cosmochim. Acta 42, 173 (1978).

${ }^{47}$ J. J. Valentini, D. P. Gerrity, D. L. Phillips, J.-C. Nieh, and K. D. Tabor, J. Chem. Phys. 86, 6745 (1987).

${ }^{48}$ J. J. Valentini, J. Chem. Phys. 86, 6757 (1987).

${ }^{49}$ S. M. Anderson, K. Mauersberger, J. Morton, and B. Schueler, ACS Symp. Ser. 502, 155 (1992); G. I. Gellene, Science 274, 1344 (1996).

${ }^{50}$ See D. R. Bates, Geophys. Res. Lett. 13, 664 (1986).

${ }^{51}$ H. Hippler, R. Rahn, and J. Troe, J. Chem. Phys. 93, 6560 (1990).

${ }^{52}$ R. A. Marcus, J. Chem. Phys. 20, 359 (1952).

${ }^{53}$ R. A. Marcus, J. Chem. Phys. 43, 2658 (1965); 52, 1018 (1970).

${ }^{54}$ P. J. Robinson and K. A. Holbrook, Unimolecular Reactions (WileyInterscience, London, 1972).

${ }^{55} \mathrm{~W}$. Forst, Theory of Unimolecular Reactions (Academic, New York, 1973).

${ }^{56}$ R. G. Gilbert and S. C. Smith, Theory of Unimolecular and Recombination Reactions (Blackwell Scientific Publications, Boston, 1990).

${ }^{57}$ T. Baer and W. L. Hase, Unimolecular Reaction Dynamics, Theory and Experiments (Oxford University Press, New York, 1996), and references therein.

${ }^{58}$ D. M. Wardlaw and R. A. Marcus, Adv. Chem. Phys. 70, 231 (1988).

${ }^{59}$ S. J. Klippenstein, in The Chemical Dynamics and Kinetics of Small Radicals, Part I, edited by K. Liu and A. Wagner (World Scientific, Singapore, 1995), and references therein.

${ }^{60}$ S. J. Klippenstein, J. Chem. Phys. 94, 6469 (1991); S. J. Klippenstein and Y.-W. Kim, ibid. 99, 5790 (1993).

${ }^{61}$ W. H. Green, Jr., C. B. Moore, and W. F. Polik, Annu. Rev. Phys. Chem. 43, 591 (1992).

${ }^{62}$ P. Pechukas and J. C. Light, J. Chem. Phys. 42, 3281 (1965).

${ }^{63}$ P. Pechukas, R. Rankin, and J. C. Light, J. Chem. Phys. 44, 794 (1966).

${ }^{64}$ J. Troe, J. Chem. Phys. 66, 4745 (1977).

${ }^{65}$ J. Troe, J. Chem. Phys. 66, 4758 (1977).

${ }^{66}$ J. Troe, J. Chem. Phys. 77, 3485 (1982). 
${ }^{67}$ W. Schranz and S. Nordholm, Int. J. Chem. Kinet. 13, 1051 (1981).

${ }^{68}$ I. Oref, J. Chem. Phys. 77, 5146 (1982).

${ }^{69}$ R. G. Gilbert, J. Chem. Phys. 80, 5501 (1984).

${ }^{70}$ J. I. Steinfeld, S. M. Alder-Golden, and J. W. Gallagher, J. Phys. Chem. Ref. Data 16, 911 (1987).

${ }^{71}$ M. R. Wiegell, N. W. Larsen, T. Pedersen, and H. Egsgaard, Int. J. Chem. Kinet. 29, 745 (1997).

${ }^{72}$ S. M. Anderson, F. S. Klein, and F. Kaufman, J. Chem. Phys. 83, 1648 (1985).

${ }^{73}$ (a) There are six unknown ${ }^{i j} \mathrm{O}_{2}$ 's, three known overall isotopic compositions $\left[{ }^{i} \mathrm{O}\right]$, and three relations $\left({ }^{i j} \mathrm{O}_{2}\right)^{2} /{ }^{i i} \mathrm{O}_{2}{ }^{j j} \mathrm{O}_{2}=\left(Q_{i j}\right)^{2} / Q_{i j} Q_{j j}$. If one tries ${ }^{i j} \mathrm{O}_{2}=\left[{ }^{i} \mathrm{O}\right]\left[{ }^{j} \mathrm{O}\right] / \Sigma_{i}\left[{ }^{i} \mathrm{O}\right]$ and ${ }^{i i} \mathrm{O}_{2}=\frac{1}{2}\left[{ }^{i} \mathrm{O}\right]^{2} / \Sigma_{i}\left[{ }^{i} \mathrm{O}\right]$, one finds that all six relations are identically satisfied when the ratio of partition functions equals four. We note that $X_{i}$ is, by definition, $\left[{ }^{i} \mathrm{O}\right] / \Sigma_{i}\left[{ }^{i} \mathrm{O}\right]$. (b) As an aside, we note that because of Eq. (4.16) it can be shown that the $K_{6, j j}^{j, 6 j}$ in Eq. (4.18) could be replaced equivalently by $4 K_{6,6 j}^{j, 66}$, though there is no advantage in doing so.

${ }^{74}$ D. W. Arnold, C. Xu, E. H. Kim, and D. Neumark, J. Chem. Phys. 101, 912 (1994)

${ }^{75}$ S. M. Anderson and K. Mauersberger, J. Geophys. Res. 100, 3033 (1995).

${ }^{76}$ A. J. Bouvier, R. Bacis, B. Bussery, S. Churassy, D. Inard, M. Notan, J. Brion, J. Malicet, and S. M. Anderson, Chem. Phys. Lett. 255, 263 (1996).

${ }^{77}$ J. Günther, S. M. Anderson, G. Hilpert, and K. Mauersberger, J. Chem. Phys. 108, 5449 (1998).

${ }^{78}$ P. Borowski, M. Fülscher, P.-A. Malmquist, and B. O. Roos, Chem. Phys. Lett. 237, 195 (1995).

${ }^{79}$ T. Tsuneda, H. Nakano, and K. Hirao, J. Chem. Phys. 103, 6520 (1995).

${ }^{80}$ A. Banichevich, S. D. Peyerimhoff, and F. Grein, Chem. Phys. 174, 93 (1993).

${ }^{81}$ D. W. Noid, M. L. Kosszykoski, and R. A. Marcus, Annu. Rev. Phys. Chem. 32, 267 (1981), and references therein.
${ }^{82}$ N. B. Slater, Theory of Unimolecular Reactions (Cornell University Press, Ithaca, 1959).

${ }^{83}$ R. A. Marcus, J. Chem. Phys. 56, 311 (1972).

${ }^{84}$ R. A. Marcus, Faraday Discuss. Chem. Soc. 75, 103 (1983), and references therein.

${ }^{85}$ P. R. Bunker, Molecular Symmetry and Spectroscopy (Academic, New York, 1979)

${ }^{86}$ S. C. Bae, H. S. Yoo, and J. K. Ku, J. Chem. Phys. 109, 1251 (1998).

${ }^{87} \mathrm{~A}$ consequence of state dependent unimolecular rate constants at any given energy is that the decay will be nonexponential, the faster rates contributing at short times, cf. R. A. Marcus, Ber. Bunsenges. Phys. Chem. 9, 209 (1988), and M. Desouter-Lecomte and F. Culot, J. Chem. Phys. 98, 7819 (1993).

${ }^{88}$ W. F. Polik, D. R. Guyer, and C. B. Moore, J. Chem. Phys. 92, 3453 (1990).

${ }^{89}$ W. H. Miller, R. Hernandez, C. B. Moore, and W. F. Polik, J. Chem. Phys. 93, 5657 (1990).

${ }^{90}$ S. M. Anderson and J. A. Kaye, Geophys. Res. Lett. 14, 91 (1987); D. R. Bates, ibid. 14, 94 (1987).

${ }^{91}$ D. R. Bates, Geophys. Res. Lett. 15, 13 (1988); D. R. Bates, J. Chem. Phys. 93, 8739 (1990).

${ }^{92}$ L. R. Khundkar, J. L. Knee, and A. H. Zewail, J. Chem. Phys. 87, 77 (1987); S. J. Klippenstein, L. R. Khundkar, A. H. Zewail, and R. A. Marcus, ibid. 89, 4761 (1988).

${ }^{93}$ E. D. Potter, M. Gruebele, L. R. Khundkar, and A. H. Zewail, Chem. Phys. Lett. 164, 463 (1989); S. J. Klippenstein and R. A. Marcus, J. Chem. Phys. 91, 2280 (1989).

${ }^{94}$ K. E. J. Hallin and A. J. Merer, J. Mol. Spectrosc. 65, 163 (1977).

${ }^{95}$ C. G. Stevens and J. C. D. Brand, J. Chem. Phys. 58, 3324 (1973).

${ }^{96}$ W. E. Howard and E. W. Schlag, Chem. Phys. 17, 123 (1976). 